\title{
Elasto-plastic strain analysis by a semi-analytical method
}

\author{
DEBABRATA DAS, PRASANTA SAHOO and KASHINATH SAHA*
}

Department of Mechanical Engineering, Jadavpur University, Kolkata 700032 e-mail: kashinathsaha@gmail.com

MS received 25 August 2007; revised 21 February 2008

\begin{abstract}
The aim of this paper is to develop a simulation model of large deformation problems following a semi-analytical method, incorporating the complications of geometric and material non-linearity in the formulation. The solution algorithm is based on the method of energy principle in structural mechanics, as applicable for conservative systems. A one-dimensional solid circular bar problem has been solved in post-elastic range assuming linear elastic, linear strain hardening material behaviour. Type of loading includes uniform uniaxial loading and gravity loading due to body force, whereas the geometry of the bar is considered to be non-uniformly taper. Results are validated successfully with benchmark solution and some new results have also been reported. The location of initiation of elasto-plastic front and its growth are found to be functions of geometry of the bar and loading conditions. Some indicative results have been presented for static and dynamic problems and the solution methodology developed for one-dimension has been extended to the elasto-plastic analysis of two-dimensional strain field problems of a rotating disk.
\end{abstract}

Keywords. Non-uniform taper bar; rotating disk; elasto-plastic strain analysis; loaded natural frequency.

\section{Introduction}

Solid slender bar is a widely used machine element found in almost every application of structural engineering. The shape and size of a bar has significant influence on its load bearing capacity. Although the conventional design of a bar, like the design of most of the machine elements, is based on the elastic region of its material, its post-elastic behaviour is also required to be addressed to make the engineering design as competitive as possible. Again the prediction of the elasto-plastic behaviour of a solid slender bar of various types of geometry as well as loading is an interesting area of work for the designers (Hill 1950).

Abdalla et al (2006) presented a simplified technique to determine the shakedown limit load of a structure using the finite element method and it was applied and verified by using two benchmark problems. Problem of two-bar structure subjected to constant axial force and cyclic thermal loading, and the three cylinder subjected to constant internal pressure and cyclic high temperature variation had been solved analytically. Yankelevsky (1999) analysed

${ }^{*}$ For correspondence 
the elasto-plastic behaviour of a shallow two-bar truss under tension or compression loading, as well as for reversal loading, to correlate the external work to the central displacement and follow the elasto-plastic stresses and strains in the bars along the loading history. Mattes \& Chimissot (1997) modelled the necking phenomenon in metallic tension members using a non-linear theory of elasto-plastic rods with deformable cross section considering the effect of the coupling between the axial deformation and the cross section deformation in tensile specimens. Kim et al (2006) performed fully plastic analyses for notched bars and plates via finite element limit analysis, based on non-hardening plasticity behaviour. Relevant geometric parameters of the notch depth and radius have been varied for observing the effect on the limit loads, and it was found that the FE solutions are significantly different from known solutions, such as slip line field solutions.

The load deflection behaviour of a uniform bar under body force loading in the postelastic region is found in the textbook of Owen \& Hinton (1980) as an example problem, and afterwards Reddy (2005) had dealt with the same problem in greater detail. Both the analyses were based on finite element method. Gadala \& Wang (1998) have used arbitrary LagrangianEulerian finite element formulation for a similar type of problem, where they had considered uniform compression of a straight bar.

Heller \& Kleiner (2006) developed a mathematical model for semi-analytical simulation of thin and thick sheet bending process. The realistic mathematical modelling of the forming process was found to be more accurate and economic than the commercial finite element analysis software, which can be utilized only in a limited way. Gang et al (2003) carried out integrity assessment of defective pipelines by using an iterative algorithm for the kinematic limit analysis of 3-D rigid-perfectly plastic bodies. The effects of various shapes and sizes of part on the plastic collapse of pipelines under internal pressure, bending moment and axial force have been investigated.

The review of available literature for axially loaded free vibration behaviour of bar indicates that no work is available on simulation of the post-elastic dynamic behaviour. Recently, Chaudhuri (2007) mentioned the importance of the research area and investigated the change in instantaneous eigen properties of a building due to yielding using perturbation approach. A related study in elastic domain were carried out by Holland et al (2008), where they investigated the free vibration behaviour of slender taper cantilever beams loaded through a cable attached to its free end using shooting method and validated the theoretical results with experimental results. Gerstmayr \& Irschik (2003) considered an elasto-plastic beam performing plane rotary motions about a fixed hinged end to describe the influence of plasticity on the vibration parameters.

Although, various researchers have carried out the elasto-plastic analysis of an axially loaded bar, the literature review indicates that research work addressing complete elastoplastic analysis by using semi-analytic method is scarce. The method has significant advantage in performance and economy when juxtaposed with the lengthy finite element method. Hence, elasto-plastic analysis of a solid circular bar of different profiles under various types of loading has been carried out and some indicative results are presented in this paper. The proposed method has also been extended successfully to analyse the elasto-plastic behaviour of a rotating disk, thus establishing its stability and robustness.

\section{Mathematical formulation}

To formulate a model for elasto-plastic material deformation three requirements have to be met (Hill 1950, Owen \& Hinton 1980); (1) An explicit description between stress and strain 


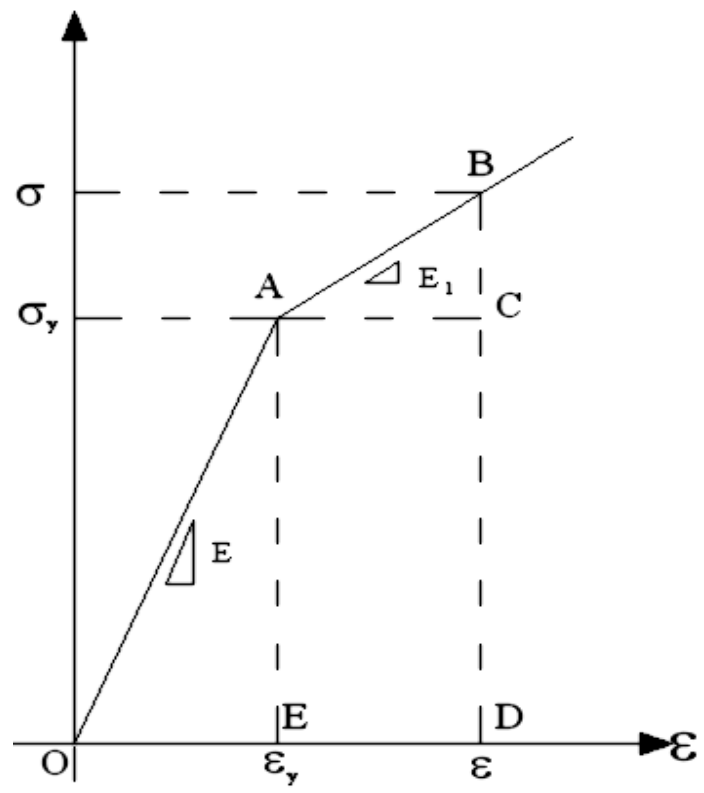

Figure 1. Linear elastic linear strain hardening elasto-plastic behaviour.

under elastic conditions; (2) A yield criterion indicating the stress level at which plastic flow commences and (3) A relationship between stress and strain when the deformation is made up of both elastic and plastic components.

Although there are various types of modelling, the present analysis is carried out for linear elastic linear strain hardening elasto-plastic material behaviour, as shown in figure 1. It is assumed that the material is isotropic and homogeneous and there is no strain-rate effect on the material behaviour. The mathematical computations of strains and stresses are based on original dimensions of the element. The problem is formulated through a variational method (Reddy 1984, Shames \& Dym 1995), where the axial displacement field is taken as unknown parameter. Assuming a series solution and using Galerkin's principle, the solution of the governing linear sets of equations is obtained.

Elasto-plastic behaviour of a linearly taper bar of solid circular cross-section under uniaxial tensile loading at the free end is solved first. The same analysis is extended for bars having exponential and parabolic variation of cross-sectional diameter. Analysis has also been carried out for increasing body force loading, and for this type of loading validation is carried out with the available results of a uniform cross section bar. Geometry of the bars for these problems is shown in figure 2 . To establish the adequacy of the present method the dynamic problem of a taper bar in elasto-plastic region has been solved and results are validated with a commercial FEM package. Finally, the method has been implemented in the two-dimensional strain field problem of a rotating axisymmetric object under centrifugal body force loading.

\subsection{Taper bar under uniaxial tensile loading}

In the present method, the classical approach for elasto-plastic analysis has not been followed strictly because that involves mathematical complexity in the formulation using variational method. Classical approach (deformation theory of plasticity) is based on the division of the total strain experienced by the loaded material into two parts, one is the 


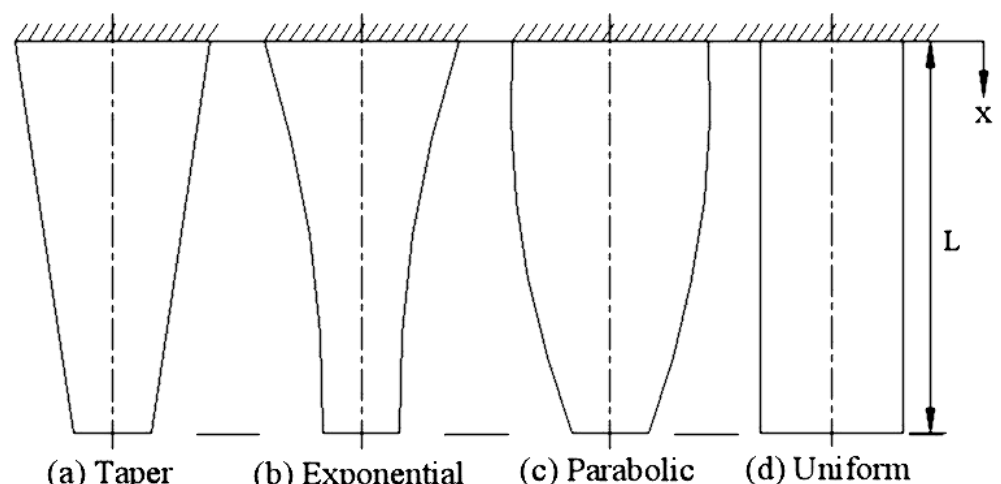

Figure 2. Geometry of bars.

elastic part (recoverable) and the other one is the plastic part (permanent). And also the constitutive equations for the plastic part are expressed in terms of suitable effective stress and effective strain related by plasticity modulus (dimensional hardening parameter), though the effective stress and effective strain become synonymous with the uniaxial stress and uniaxial strain respectively for one-dimensional problems.

The present approach involves calculation of strain energy which is the area under stressstrain diagram and that is why the total strain is divided into two parts, one is that corresponding to initiation of uniaxial yielding ( $\varepsilon_{y}$ in figure 1 ) and the other one is remaining part of total strain $\left(\varepsilon-\varepsilon_{y}\right)$ in figure 1 . Accordingly, the area OAE in figure 1 is termed as elastic part of strain energy and the area $\mathrm{ABDE}$ in figure 1 is termed as post-elastic part of strain energy. In this approach, the terms 'elastic' and 'post-elastic' are not to be mistaken with the terms 'elastic' and 'plastic' used in the classical approach. One similarity between the present approach and deformation theory of plasticity is that both are based on total strain formulation as compared to the flow theory, which is based on incremental strain. So, it can be said that the present method is an alternative approach to classical one leading to same end result and is quite simple and robust. It is worthwhile to mention here that the concept of division of total strain by the present approach is equally valid for two-dimensional problems as discussed in $\S 5$, but the strain corresponding to initiation of yielding is not same as uniaxial yield strain and for that a separate algorithm based on iterative procedure has been developed. It is to be noted further that the effect of change in geometry due to presence of plastic deformation is not taken into account in the mathematical formulation of the present method.

The solution for the elasto-plastic displacement field of a body under equilibrium is obtained from an extension of the minimum potential energy principle,

$$
\delta(U+V)=0,
$$

where, $U\left(=U_{e}+U_{p}\right)$ is the total strain energy, consisting of an elastic $\left(U_{e}\right)$ and a postelastic $\left(U_{p}\right)$ part and $V$ is the potential energy developed by the external forces. It is assumed that principle of minimum potential energy remains valid for bilinear elastic material, unless one considers a cyclic loading problem. For uniform loading and taper geometry, yielding initiates from the free end and gradually proceeds towards the fixed end. Let, at any instant, the interface between the elastic and the post-elastic region is at a distance $L_{e}$ from the fixed end. The notations have been indicated in detail in figure 3 and in addition, a list of notations is given. 


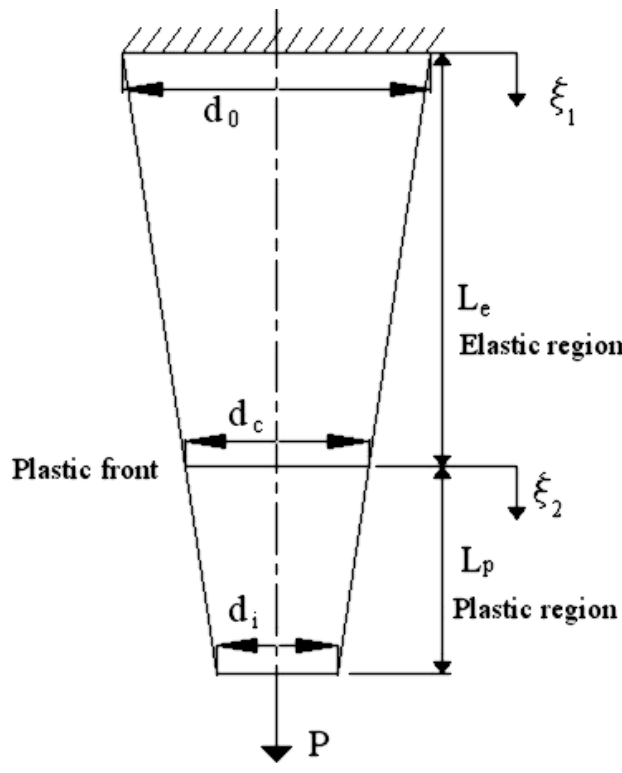

(i) Notations for uniform axial loading

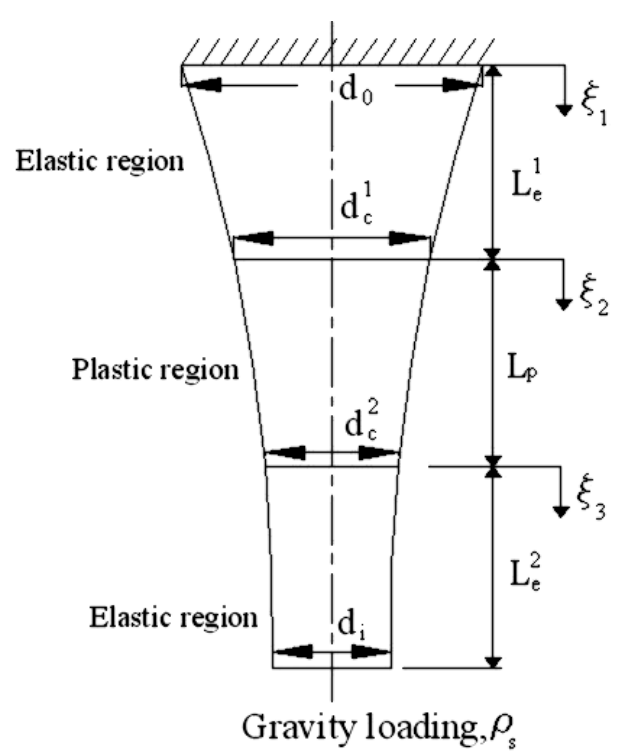

(ii) Notations for gravity loading due to body force

Figure 3. Graphical representations of nomenclatures.

$d_{0}, d_{i}$ and $d_{c}$ are diameter of the bar at the fixed end, free end and elasto-plastic interface respectively; $L, L_{e}$ and $L_{p}$ are total length, length of the elastic region and length of the plastic region respectively. The diameter $d$ at any distance $x$ from the fixed end is given by,

$d=d_{0}-\frac{x}{L}\left(d_{0}-d_{i}\right)=d_{0}-\xi\left(d_{0}-d_{i}\right)=d_{0}-\xi_{1}\left(d_{0}-d_{c}\right)=d_{c}-\xi_{2}\left(d_{c}-d_{i}\right)$,

where $\xi$ is the normalized global coordinate corresponding to $x$ and $\xi_{1}$ and $\xi_{2}$ are normalized local coordinates in the elastic region and in the plastic region respectively. Thus,

$$
\xi=x / L, \xi_{1}=x / L_{e} \text { and } \xi_{2}=\left(x-L_{e}\right) / L_{p}
$$

The diameter at the location of plastic front corresponding to any load $P$ can be obtained by the relation, $d_{c}=\sqrt{4 P /\left(\pi \sigma_{y}\right)}$, where $\sigma_{y}$ is the yield strength of the bar material. Then, the length $L_{e}$ can be obtained using equations (2) and (3).

For a uniaxially loaded taper bar, the variation in normal stress between two adjacent layers would give rise to shear stresses as well, but their contribution in the strain energy has been neglected, being insignificant. Regarding strain-displacement relation, instead of the linear one $\left(\varepsilon_{x}=\frac{d u}{d x}\right)$, a more accurate non-linear expression $\left(\varepsilon_{x}=\frac{d u}{d x}+\frac{1}{2}\left(\frac{d u}{d x}\right)^{2}\right)$ has been used. With these assumptions the expressions of elastic and post-elastic part of strain energy are,

$$
U_{e}=\frac{\pi E}{8} \int_{0}^{L_{e}} d^{2}\left\{\frac{d u}{d x}+\frac{1}{2}\left(\frac{d u}{d x}\right)^{2}\right\}^{2} d x
$$


and

$$
\begin{aligned}
U_{p}= & \frac{\pi E_{1}}{32} \int_{L_{e}}^{L} d^{2}\left(\frac{d u}{d x}\right)^{4} d x+\frac{\pi E_{1}}{8} \int_{L_{e}}^{L} d^{2}\left(\frac{d u}{d x}\right)^{3} d x \\
& +\frac{\pi\left\{E_{1}+\varepsilon_{y}\left(E-E_{1}\right)\right\}}{8} \int_{L_{e}}^{L} d^{2}\left(\frac{d u}{d x}\right)^{2} d x \\
& +\frac{\pi\left(E-E_{1}\right)}{4} \varepsilon_{y} \int_{L_{e}}^{L} d^{2}\left(\frac{d u}{d x}\right) d x-\frac{\pi\left(E-E_{1}\right)}{8} \varepsilon_{y}^{2} \int_{L_{e}}^{L} d^{2} d x,
\end{aligned}
$$

where, $u$ is the displacement field; $E$ and $E_{1}$ are elastic modulus and tangent modulus of the bar material; $\sigma_{y}$ and $\varepsilon_{y}$ are stress and strain corresponding to yield position of the bar material. Again, the expression for work potential is given by

$$
V=-P \int_{0}^{L_{e}}\left\{\frac{d u}{d x}+\frac{1}{2}\left(\frac{d u}{d x}\right)^{2}\right\} d x-P \int_{L_{e}}^{L}\left\{\frac{d u}{d x}+\frac{1}{2}\left(\frac{d u}{d x}\right)^{2}\right\} d x
$$

Substituting the normalized form of equations (4), (5) and (6) in equation (1), the governing equation is obtained as

$$
\begin{aligned}
& \frac{\pi E}{8} \int_{0}^{1}\left(\frac{1}{L_{e}} \frac{d u}{d \xi_{1}}\right)^{2} d^{2}\left(\frac{d u}{d \xi_{1}}\right) \delta\left(\frac{d u}{d \xi_{1}}\right) d^{2} d \xi_{1}+\frac{3 \pi E}{8} \int_{0}^{1}\left(\frac{1}{L_{e}} \frac{d u}{d \xi_{1}}\right) d^{2}\left(\frac{d u}{d \xi_{1}}\right) \delta\left(\frac{d u}{d \xi_{1}}\right) d \xi_{1} \\
&+\frac{\pi E}{4} \int_{0}^{1} d^{2}\left(\frac{d u}{d \xi_{1}}\right) \delta\left(\frac{d u}{d \xi_{1}}\right) d \xi_{1}+\frac{\pi E_{1}}{8} \int_{0}^{1}\left(\frac{1}{L_{p}} \frac{d u}{d \xi_{2}}\right)^{2} d^{2}\left(\frac{d u}{d \xi_{2}}\right) \delta\left(\frac{d u}{d \xi_{2}}\right) d \xi_{2} \\
&+\frac{3 \pi E_{1}}{8} \int_{0}^{1}\left(\frac{1}{L_{p}} \frac{d u}{d \xi_{2}}\right) d^{2}\left(\frac{d u}{d \xi_{2}}\right) \delta\left(\frac{d u}{d \xi_{2}}\right) d \xi_{2} \\
&+\frac{\pi\left\{E_{1}+\varepsilon_{y}\left(E-E_{1}\right)\right\}}{4} \int_{0}^{1} d^{2}\left(\frac{d u}{d \xi_{2}}\right) \delta\left(\frac{d u}{d \xi_{2}}\right) d \xi_{2} \\
& \quad-\frac{P}{L_{e}} \int_{0}^{1}\left(\frac{d u}{d \xi_{1}}\right) \delta\left(\frac{d u}{d \xi_{1}}\right) d \xi_{1}-\frac{P}{L_{p}} \int_{0}^{1}\left(\frac{d u}{d \xi_{2}}\right) \delta\left(\frac{d u}{d \xi_{2}}\right) d \xi_{2} \\
&= P \int_{0}^{1} \delta\left(\frac{d u}{d \xi_{1}}\right) d \xi_{1}+P \int_{0}^{1} \delta\left(\frac{d u}{d \xi_{2}}\right) d \xi_{2}-\frac{\pi\left(E-E_{1}\right)}{4} \varepsilon_{y} \int_{0}^{1} d^{2} \delta\left(\frac{d u}{d \xi_{2}}\right) d \xi_{2} .
\end{aligned}
$$

The global displacement function $u(\xi)$ in equation (7) may be approximated by

$$
u(\xi) \cong \sum c_{i} \phi_{i}, i=1,2, \ldots, n f
$$

where $\phi_{i}$ is the set of orthogonal functions developed through Gram-Schmidt scheme. The necessary starting function to generate the higher order orthogonal functions is selected by satisfying the relevant geometric boundary conditions, i.e. $u=0$ at $\xi=0$. Displacement functions in the elastic and post-elastic regions are expressed as $u\left(\xi_{1}\right) \cong \sum c_{i} \phi_{i}{ }^{e}$ and 
$u\left(\xi_{2}\right) \cong \sum c_{i} \phi_{i}{ }^{p}$ respectively. Substituting these assumed displacement functions and replacing operator $\delta$ by $\partial / \partial c_{j}$ the governing equation is obtained in matrix form, and is given as

$$
\begin{aligned}
& \frac{\pi E}{8 L_{e}} \sum_{j=1}^{n f} \sum_{i=1}^{n f} c_{i} \int_{0}^{1}\left(\sum_{i=1}^{n f} c_{i} \phi_{i}^{e}\right)^{2} d^{2}\left(\frac{\partial \phi_{i}^{e}}{\partial \xi_{1}}\right)\left(\frac{\partial \phi_{j}^{e}}{\partial \xi_{1}}\right) d \xi_{1} \\
& +\frac{3 \pi E}{8 L_{e}} \sum_{j=1}^{n f} \sum_{i=1}^{n f} c_{i} \int_{0}^{1}\left(\sum_{i=1}^{n f} c_{i} \phi_{i}^{e}\right) d^{2}\left(\frac{\partial \phi_{i}^{e}}{\partial \xi_{1}}\right)\left(\frac{\partial \phi_{j}^{e}}{\partial \xi_{1}}\right) d \xi_{1} \\
& +\frac{\pi E}{4 L_{e}} \sum_{j=1}^{n f} \sum_{i=1}^{n f} c_{i} \int_{0}^{1} d^{2}\left(\frac{\partial \phi_{i}^{e}}{\partial \xi_{1}}\right)\left(\frac{\partial \phi_{j}^{e}}{\partial \xi_{1}}\right) d \xi_{1} \\
& +\frac{\pi E_{1}}{8 L_{p}} \sum_{j=1}^{n f} \sum_{i=1}^{n f} c_{i} \int_{0}^{1}\left(\sum_{i=1}^{n f} c_{i} \phi_{i}^{p}\right)^{2} d^{2}\left(\frac{\partial \phi_{i}^{p}}{\partial \xi_{2}}\right)\left(\frac{\partial \phi_{j}^{p}}{\partial \xi_{2}}\right) d \xi_{2} \\
& +\frac{3 \pi E_{1}}{8 L_{p}} \sum_{j=1}^{n f} \sum_{i=1}^{n f} c_{i} \int_{0}^{1}\left(\sum_{i=1}^{n f} c_{i} \phi_{i}^{p}\right) d^{2}\left(\frac{\partial \phi_{i}^{p}}{\partial \xi_{2}}\right)\left(\frac{\partial \phi_{j}^{p}}{\partial \xi_{2}}\right) d \xi_{2} \\
& +\frac{\pi\left\{E_{1}+\varepsilon_{y}\left(E-E_{1}\right)\right\}}{4 L_{p}} \sum_{j=1}^{n f} \sum_{i=1}^{n f} c_{i} \int_{0}^{1} d^{2}\left(\frac{\partial \phi_{i}^{p}}{\partial \xi_{2}}\right)\left(\frac{\partial \phi_{j}^{p}}{\partial \xi_{2}}\right) d \xi_{2} \\
& -\frac{P}{L_{e}} \sum_{j=1}^{n f} \sum_{i=1}^{n f} c_{i} \int_{0}^{1}\left(\frac{\partial \phi_{i}^{e}}{\partial \xi_{1}}\right)\left(\frac{\partial \phi_{j}^{e}}{\partial \xi_{1}}\right) d \xi_{1}-\frac{P}{L_{p}} \sum_{j=1}^{n f} \sum_{i=1}^{n f} c_{i} \int_{0}^{1}\left(\frac{\partial \phi_{i}^{p}}{\partial \xi_{2}}\right)\left(\frac{\partial \phi_{j}^{p}}{\partial \xi_{2}}\right) d \xi_{2} \\
& =P \sum_{j=1}^{n f} \int_{0}^{1}\left(\frac{\partial \phi_{j}^{e}}{\partial \xi_{1}}\right) d \xi_{1}+P \sum_{j=1}^{n f} \int_{0}^{1}\left(\frac{\partial \phi_{j}^{p}}{\partial \xi_{2}}\right) d \xi_{2}-\frac{\pi\left(E-E_{1}\right)}{4} \varepsilon_{y} \sum_{j=1}^{n f} \int_{0}^{1} d^{2}\left(\frac{\partial \phi_{j}^{p}}{\partial \xi_{2}}\right) d \xi_{2} .
\end{aligned}
$$

The set of equations in (8) are non-linear in nature, which is solved by direct substitution method using successive relaxation parameter.

\subsection{Exponential and parabolic bar under uniaxial loading}

The exponential and parabolic variation in cross-sectional diameter with the axial coordinate are given by the following equations

$$
\begin{aligned}
& d=d_{0} \exp \left(-n \xi^{k}\right) \\
& d=d_{0}\left(1-n \xi^{k}\right)
\end{aligned}
$$

where, $n$ and $k$ are the parameters governing the geometry. The value of $n$ is always selected so as to make the diameter largest at the fixed end. Accordingly, yielding initiates from the free end and gradually proceeds towards the fixed end with increasing load. The distance of 
the elasto-plastic interface from the fixed end, $L_{e}$ is obtained for exponential and parabolic variations by rearranging equations (9) and (10) as follows:

$$
\begin{aligned}
& L_{e}=\left\{-\frac{1}{n} \ln \left(\frac{d_{c}}{d_{0}}\right)\right\}^{\frac{1}{k}} \\
& L_{e}=\left\{\frac{1}{n}\left(1-\frac{d_{c}}{d_{0}}\right)\right\}^{\frac{1}{k}},
\end{aligned}
$$

where $d_{c}$ is the diameter at the location of plastic front at any load $P$. The formulation and other nomenclature given for taper bar problem remain same for the non-uniform problem and hence the governing equation for this problem is also expressed by equation (8).

\subsection{Bar under gravity loading}

For this type of loading, unlike the previous problems, yielding may initiate at the fixed end and gradually proceed towards the free end with increasing body force or it may initiate at any intermediate axial location and gradually proceed both ways, depending on the geometry of the bar. The normalized form of the expression of work potential for the case when yielding initiates at the fixed end is given by,

$$
\begin{aligned}
V= & -\frac{\pi \rho_{s}}{4} \int_{0}^{1}\left(L-\xi_{1} L_{p}\right) d^{2}\left(\frac{d u}{d \xi_{1}}\right) d \xi_{1}-\frac{\pi \rho_{s}}{8 L_{p}} \int_{0}^{1}\left(L-\xi_{1} L_{p}\right) d^{2}\left(\frac{d u}{d \xi_{1}}\right)^{2} d \xi_{1} \\
& -\frac{\pi \rho_{s}}{4} \int_{0}^{1}\left(L_{e}-\xi_{2} L_{e}\right) d^{2}\left(\frac{d u}{d \xi_{2}}\right) d \xi_{2}-\frac{\pi \rho_{s}}{8 L_{e}} \int_{0}^{1}\left(L_{e}-\xi_{2} L_{e}\right) d^{2}\left(\frac{d u}{d \xi_{2}}\right) d \xi_{2}
\end{aligned}
$$

where, $\rho_{s}$ is the specific weight of the bar material at any instant; $A$ is the cross-sectional area at any general axial location; $\xi_{1}$ and $\xi_{2}$ are normalized local coordinate in the plastic region and elastic region respectively.

The location of the elasto-plastic interface from the fixed end is obtained numerically by an iterative method. Here the notations $L_{e}$ and $L_{p}$ do not follow figure 3(i), rather they interchange there positions and accordingly $\xi_{1}$ and $\xi_{2}$ have been redefined, as shown in figure 3(ii) and indicated in the list of notations.

Using the formulation of the taper bar problem, the governing differential equation for the present case is obtained in matrix form as follows:

$$
\begin{aligned}
\frac{\pi E}{8 L_{e}} \sum_{j=1}^{n f} \sum_{i=1}^{n f} c_{i} \int_{0}^{1}\left(\sum_{i=1}^{n f} c_{i} \phi_{i}^{e}\right)^{2} d^{2}\left(\frac{\partial \phi_{i}^{e}}{\partial \xi_{2}}\right)\left(\frac{\partial \phi_{j}^{e}}{\partial \xi_{2}}\right) d \xi_{2} \\
+\frac{3 \pi E}{8 L_{e}} \sum_{j=1}^{n f} \sum_{i=1}^{n f} c_{i} \int_{0}^{1}\left(\sum_{i=1}^{n f} c_{i} \phi_{i}^{e}\right) d^{2}\left(\frac{\partial \phi_{i}^{e}}{\partial \xi_{2}}\right)\left(\frac{\partial \phi_{j}^{e}}{\partial \xi_{2}}\right) d \xi_{2} \\
+\frac{\pi E}{4 L_{e}} \sum_{j=1}^{n f} \sum_{i=1}^{n f} c_{i} \int_{0}^{1} d^{2}\left(\frac{\partial \phi_{i}^{e}}{\partial \xi_{2}}\right)\left(\frac{\partial \phi_{j}^{e}}{\partial \xi_{2}}\right) d \xi_{2}
\end{aligned}
$$




$$
\begin{aligned}
& +\frac{\pi E_{1}}{8 L_{p}} \sum_{j=1}^{n f} \sum_{i=1}^{n f} c_{i} \int_{0}^{1}\left(\sum_{i=1}^{n f} c_{i} \phi_{i}^{p}\right)^{2} d^{2}\left(\frac{\partial \phi_{i}^{p}}{\partial \xi_{1}}\right)\left(\frac{\partial \phi_{j}^{p}}{\partial \xi_{1}}\right) d \xi_{1} \\
& +\frac{3 \pi E_{1}}{8 L_{p}} \sum_{j=1}^{n f} \sum_{i=1}^{n f} c_{i} \int_{0}^{1}\left(\sum_{i=1}^{n f} c_{i} \phi_{i}^{p}\right) d^{2}\left(\frac{\partial \phi_{i}^{p}}{\partial \xi_{1}}\right)\left(\frac{\partial \phi_{j}^{p}}{\partial \xi_{1}}\right) d \xi_{1} \\
& +\frac{\pi\left\{E_{1}+\varepsilon_{y}\left(E-E_{1}\right)\right\}}{4 L_{p}} \sum_{j=1}^{n f} \sum_{i=1}^{n f} c_{i} \int_{0}^{1} d^{2}\left(\frac{\partial \phi_{i}^{p}}{\partial \xi_{1}}\right) \delta\left(\frac{\partial \phi_{j}^{p}}{\partial \xi_{1}}\right) d \xi_{1} \\
& -\frac{\pi \rho_{s}}{4 L_{e}} \sum_{j=1}^{n f} \sum_{i=1}^{n f} c_{i} \int_{0}^{1}\left(L_{e}-\xi_{2} L_{e}\right) d^{2}\left(\frac{\partial \phi_{i}^{e}}{\partial \xi_{2}}\right)\left(\frac{\partial \phi_{j}^{e}}{\partial \xi_{2}}\right) d \xi_{2} \\
& -\frac{\pi \rho_{s}}{4 L_{p}} \sum_{j=1}^{n f} \sum_{i=1}^{n f} c_{i} \int_{0}^{1}\left(L-\xi_{1} L_{p}\right) d^{2}\left(\frac{\partial \phi_{i}^{p}}{\partial \xi_{1}}\right)\left(\frac{\partial \phi_{j}^{p}}{\partial \xi_{1}}\right) d \xi_{1} \\
& =\frac{\pi \rho_{s}}{4} \sum_{j=1}^{n f} \int_{0}^{1}\left(L_{e}-\xi_{2} L_{e}\right) d^{2}\left(\frac{\partial \phi_{j}^{e}}{\partial \xi_{2}}\right) d \xi_{2}+\frac{\pi \rho_{s}}{4} \sum_{j=1}^{n f} \int_{0}^{1}\left(L-\xi_{1} L_{p}\right) d^{2}\left(\frac{\partial \phi_{j}^{p}}{\partial \xi_{1}}\right) d \xi_{1} \\
& -\frac{\pi\left(E-E_{1}\right)}{4} \varepsilon_{y} \sum_{j=1}^{n f} \int_{0}^{1} d^{2}\left(\frac{\partial \phi_{j}^{p}}{\partial \xi_{1}}\right) d \xi_{1} .
\end{aligned}
$$

The work potential for the case when yielding initiates at any location in between the two end sections is given by,

$$
\begin{aligned}
V= & -\frac{\pi \rho_{s}}{4} \int_{0}^{1}\left(L-\xi_{1} L_{e}^{1}\right) d^{2}\left(\frac{\partial u}{\partial \xi_{1}}\right) d \xi_{1}-\frac{\pi \rho_{s}}{8 L_{e}^{1}} \int_{0}^{1}\left(L-\xi_{1} L_{e}^{1}\right) d^{2}\left(\frac{\partial u}{\partial \xi_{1}}\right)^{2} d \xi_{1} \\
& -\frac{\pi \rho_{s}}{4} \int_{0}^{1}\left(L-L_{e}^{1}-\xi_{2} L_{p}\right) d^{2}\left(\frac{\partial u}{\partial \xi_{2}}\right) d \xi_{2}-\frac{\pi \rho_{s}}{8 L_{p}} \int_{0}^{1}\left(L-L_{e}^{1}-\xi_{2} L_{p}\right) d^{2}\left(\frac{\partial u}{\partial \xi_{2}}\right)^{2} d \xi_{2} \\
& -\frac{\pi \rho_{s}}{4} \int_{0}^{1}\left(L_{e}^{2}-\xi_{3} L_{e}^{2}\right) d^{2}\left(\frac{\partial u}{\partial \xi_{3}}\right) d \xi_{3}-\frac{\pi \rho_{s}}{8 L_{e}^{2}} \int_{0}^{1}\left(L_{e}^{2}-\xi_{3} L_{e}^{2}\right) d^{2}\left(\frac{\partial u}{\partial \xi_{3}}\right)^{2} d \xi_{3},
\end{aligned}
$$

where, $L_{e}^{1}$ and $L_{e}^{2}$ are lengths of two elastic regions attached to fixed and free end respectively; $\xi_{1}, \xi_{2}$ and $\xi_{3}$ are normalized local coordinates corresponding to elastic region attached to fixed end, plastic region at the mid-region and elastic region attached to free end respectively. These notations have also been indicated in figure 3(ii) for clarity. The governing equation for this case is given as,

$$
\begin{aligned}
& \frac{\pi E}{8 L_{e}^{1}} \sum_{j=1}^{n f} \sum_{i=1}^{n f} c_{i} \int_{0}^{1}\left(\frac{1}{L_{e}^{1}} \sum_{i=1}^{n f} c_{i} \phi_{i}^{e 1}\right)^{2} d^{2}\left(\frac{\partial \phi_{i}^{e 1}}{\partial \xi_{1}}\right)\left(\frac{\partial \phi_{j}^{e 1}}{\partial \xi_{1}}\right) d \xi_{1} \\
& \quad+\frac{3 \pi E}{8 L_{e}^{1}} \sum_{j=1}^{n f} \sum_{i=1}^{n f} c_{i} \int_{0}^{1}\left(\frac{1}{L_{e}^{1}} \sum_{i=1}^{n f} c_{i} \phi_{i}^{e 1}\right) d^{2}\left(\frac{\partial \phi_{i}^{e 1}}{\partial \xi_{1}}\right)\left(\frac{\partial \phi_{j}^{e 1}}{\partial \xi_{1}}\right) d \xi_{1}
\end{aligned}
$$




$$
\begin{aligned}
& +\frac{\pi E}{4 L_{e}^{1}} \sum_{j=1}^{n f} \sum_{i=1}^{n f} c_{i} \int_{0}^{1} d^{2}\left(\frac{\partial \phi_{i}^{e 1}}{\partial \xi_{1}}\right)\left(\frac{\partial \phi_{j}^{e 1}}{\partial \xi_{1}}\right) d \xi_{1} \\
& +\frac{\pi E_{1}}{8 L_{p}} \sum_{j=1}^{n f} \sum_{i=1}^{n f} c_{i} \int_{0}^{1}\left(\frac{1}{L_{p}} \sum_{i=1}^{n f} c_{i} \phi_{i}^{p}\right)^{2} d^{2}\left(\frac{\partial \phi_{i}^{p}}{\partial \xi_{2}}\right)\left(\frac{\partial \phi_{j}^{p}}{\partial \xi_{2}}\right) d \xi_{2} \\
& +\frac{3 \pi E_{1}}{8 L_{p}} \sum_{j=1}^{n f} \sum_{i=1}^{n f} c_{i} \int_{0}^{1}\left(\frac{1}{L_{p}} \sum_{i=1}^{n f} c_{i} \phi_{i}^{p}\right) d^{2}\left(\frac{\partial \phi_{i}^{p}}{\partial \xi_{2}}\right)\left(\frac{\partial \phi_{j}^{p}}{\partial \xi_{2}}\right) d \xi_{2} \\
& +\frac{\pi\left\{E_{1}+\varepsilon_{y}\left(E-E_{1}\right)\right\}}{4 L_{p}} \sum_{j=1}^{n f} \sum_{i=1}^{n f} c_{i} \int_{0}^{1} d^{2}\left(\frac{\partial \phi_{i}^{p}}{\partial \xi_{2}}\right)\left(\frac{\partial \phi_{j}^{p}}{\partial \xi_{2}}\right) d \xi_{2} \\
& +\frac{\pi E}{8 L_{e}^{2}} \sum_{j=1}^{n f} \sum_{i=1}^{n f} c_{i} \int_{0}^{1}\left(\frac{1}{L_{e}^{2}} \sum_{i=1}^{n f} c_{i} \phi_{i}^{e 2}\right)^{2} d^{2}\left(\frac{\partial \phi_{i}^{e 2}}{\partial \xi_{3}}\right)\left(\frac{\partial \phi_{j}^{e 2}}{\partial \xi_{3}}\right) d \xi_{3} \\
& +\frac{3 \pi E}{8 L_{e}^{2}} \sum_{j=1}^{n f} \sum_{i=1}^{n f} c_{i} \int_{0}^{1}\left(\frac{1}{L_{e}^{2}} \sum_{i=1}^{n f} c_{i} \phi_{i}^{e 2}\right) d^{2}\left(\frac{\partial \phi_{i}^{e 2}}{\partial \xi_{3}}\right)\left(\frac{\partial \phi_{j}^{e 2}}{\partial \xi_{3}}\right) d \xi_{3} \\
& +\frac{\pi E}{4 L_{e}^{2}} \sum_{j=1}^{n f} \sum_{i=1}^{n f} c_{i} \int_{0}^{1} d^{2}\left(\frac{\partial \phi_{i}^{e 2}}{\partial \xi_{3}}\right)\left(\frac{\partial \phi_{j}^{e 2}}{\partial \xi_{3}}\right) d \xi_{3} \\
& -\frac{\pi \rho_{s}}{4 L_{e}^{1}} \sum_{j=1}^{n f} \sum_{i=1}^{n f} c_{i} \int_{0}^{1}\left(L-\xi_{1} L_{e}^{1}\right) d^{2}\left(\frac{\partial \phi_{i}^{e 1}}{\partial \xi_{1}}\right)\left(\frac{\partial \phi_{j}^{e 1}}{\partial \xi_{1}}\right) d \xi_{1} \\
& -\frac{\pi \rho_{s}}{4 L_{p}} \sum_{j=1}^{n f} \sum_{i=1}^{n f} c_{i} \int_{0}^{1}\left(L-L_{e}^{1}-\xi_{2} L_{p}\right) d^{2}\left(\frac{\partial \phi_{i}^{p}}{\partial \xi_{2}}\right)\left(\frac{\partial \phi_{j}^{p}}{\partial \xi_{2}}\right) d \xi_{2} \\
& -\frac{\pi \rho_{s}}{4 L_{e}^{2}} \sum_{j=1}^{n f} \sum_{i=1}^{n f} c_{i} \int_{0}^{1}\left(L_{e}^{2}-\xi_{3} L_{e}^{2}\right) d^{2}\left(\frac{\partial \phi_{i}^{e 2}}{\partial \xi_{3}}\right)\left(\frac{\partial \phi_{j}^{e 2}}{\partial \xi_{3}}\right) d \xi_{3} \\
& =\frac{\pi \rho_{s}}{4} \sum_{j=1}^{n f} \int_{0}^{1}\left(L-\xi_{1} L_{e}^{1}\right) d^{2}\left(\frac{\partial \phi_{j}^{e 1}}{\partial \xi_{1}}\right) d \xi_{1} \\
& +\frac{\pi \rho_{s}}{4} \sum_{j=1}^{n f} \int_{0}^{1}\left(L-L_{e}^{1}-\xi_{2} L_{p}\right) d^{2}\left(\frac{\partial \phi_{j}^{p}}{\partial \xi_{2}}\right) d \xi_{2} \\
& +\frac{\pi \rho_{s}}{4} \sum_{j=1}^{n f} \int_{0}^{1}\left(L_{e}^{2}-\xi_{3} L_{e}^{2}\right) d^{2}\left(\frac{\partial \phi_{j}^{e 2}}{\partial \xi_{3}}\right) d \xi_{3} \\
& -\frac{\pi\left(E-E_{1}\right)}{4} \varepsilon_{y} \sum_{j=1}^{n f} \int_{0}^{1} d^{2}\left(\frac{\partial \phi_{j}^{p}}{\partial \xi_{2}}\right) d \xi_{2}
\end{aligned}
$$

where $\phi_{i}^{e 1}$ and $\phi_{i}^{e 2}$ are the local displacement functions in the elastic regions attached to the fixed and free ends respectively. 


\section{Results and discussions}

The load at which yielding initiates is called elastic limit load $\left(P_{y}\right.$ or $\left.\rho_{s y}\right)$. The load-deflection characteristics have been presented for different geometry and loading conditions through the plots for maximum normalized deflection $\left(u^{*}=u_{1} / L\right)$ against normalized load $\left(P^{*}=\right.$ $P / P_{y}$ or, $\left.P^{*}=\rho_{s} / \rho_{s y}\right)$. Corresponding to all these plots, the plots for normalized plastic front location $\left(x^{*}=x_{p} / L\right)$ have been provided to capture the advancement of plastic front with increasing load. Here, $u_{1}$ denotes the maximum deflection at the free end of the bar and $x_{p}$ represents the dimensional coordinate of the location of the plastic front. Results have been generated with the following values of material and geometric parameters: $E=$ $210 \mathrm{GPa}, E_{1}=70 \mathrm{GPa}, d_{0}=0.1 \mathrm{~m}$ and $L=1 \mathrm{~m}$.

\subsection{Validation of the present approach}

To check the suitability and validity of the present approach, two comparison problems are considered; one for a linearly taper bar under uniaxial tension and the other for a solid circular bar under body force loading. A comparative study has been carried out between the present method and analysis with commercial finite element package ANSYS (version 8.0) using the element BEAM 188 for a linearly taper bar with $d_{i}=0.025 \mathrm{~m}$. The validation plot for $P^{*}$ vs $u^{*}$ given in figure 4(i) shows that the agreement of present results with the same using ANSYS is excellent and the correctness of the present formulation is established for analysis of bar under uniaxial tension. As a second comparison, the plot for reaction force vs displacement at the free end for a solid circular bar under body force loading has been validated with that given in Reddy (2005), as shown in figure 4(ii), indicating good agreement. The parameter values used for generating this plot are mentioned in the figure.

\subsection{Bar under uniaxial tension}

Characteristic plots of normalized load vs displacement for a linearly taper bar have been obtained by varying the free end diameter of the bar and are shown in figure 5 (a). The corresponding plots for normalized load vs plastic front location have been furnished in



(i) Linearly taper bar under uniaxial tension

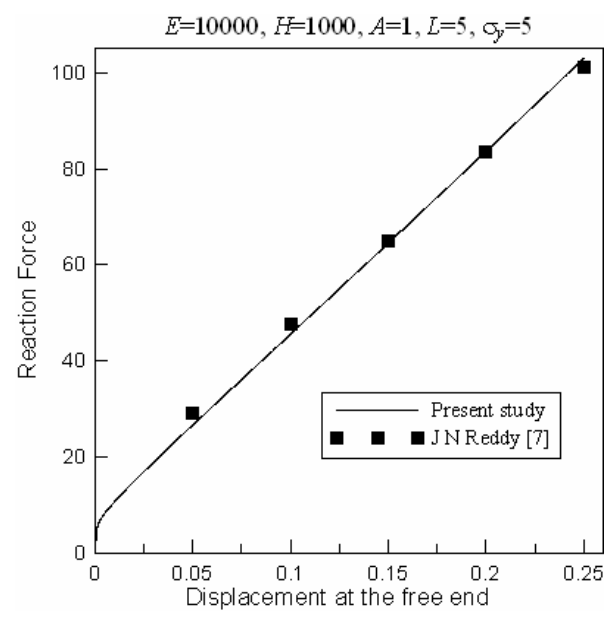

(ii) Uniform solid bar under body force loading

Figure 4. Validation plots. 

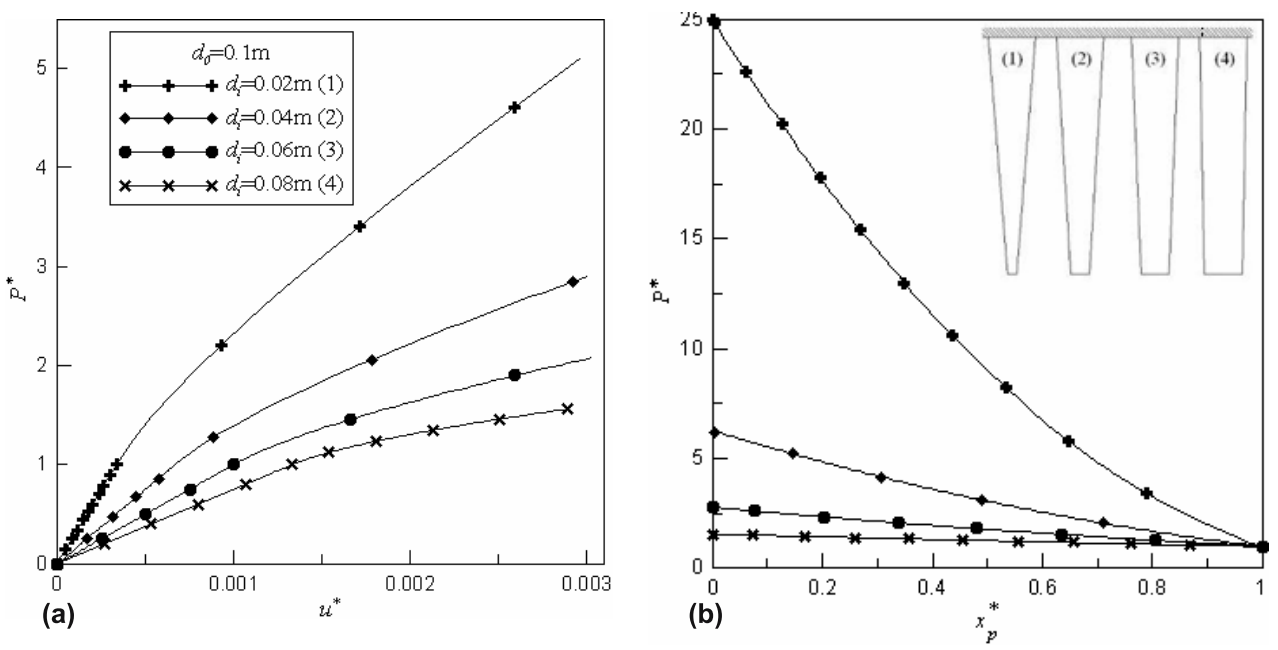

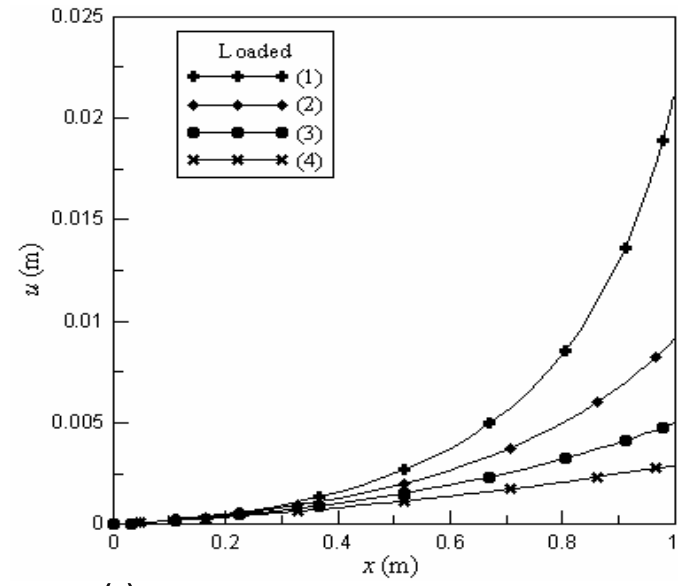

(c)



(d)

Figure 5. Plot for linearly taper bar under uniaxial tensile loading: (a) $P^{*}$ vs $u^{*}$, (b) $P^{*}$ vs $x^{*}$, (c) $u$ vs $x$ corresponding to collapse loading and (d) $u$ vs $x$ after unloading.

figure 5 (b). In the first figure, the geometry of the bar has been represented by numerical values whereas in the second one, the shapes have been indicated graphically. It is to be noted that the extent of loading is not identical in the two cases. In plot (b) loading is continued up to the collapse load $P_{c}$, i.e. the load corresponding to which the total bar attains postelastic state. However, in plot (a) loading has been restricted to maintain better clarity. The displacement field in the bars corresponding to collapse load is shown in figure 5(c) and the residual displacement field after unloading from the collapse load is shown in figure 5(d). The permanent deformation in the bars can be easily obtained from these two figures. The residual displacement field is obtained through an elastic unloading from the collapse load following linear elastic material behaviour with elastic modulus $E$. The actual implementation involves subtraction of the linear displacement field from the actual one, obtained through bi-linear elastic analysis. Plots for non-uniform taper bars having exponential and parabolic variation are shown in figures 6 and 7 respectively. The sequence of plots and conventions used in 

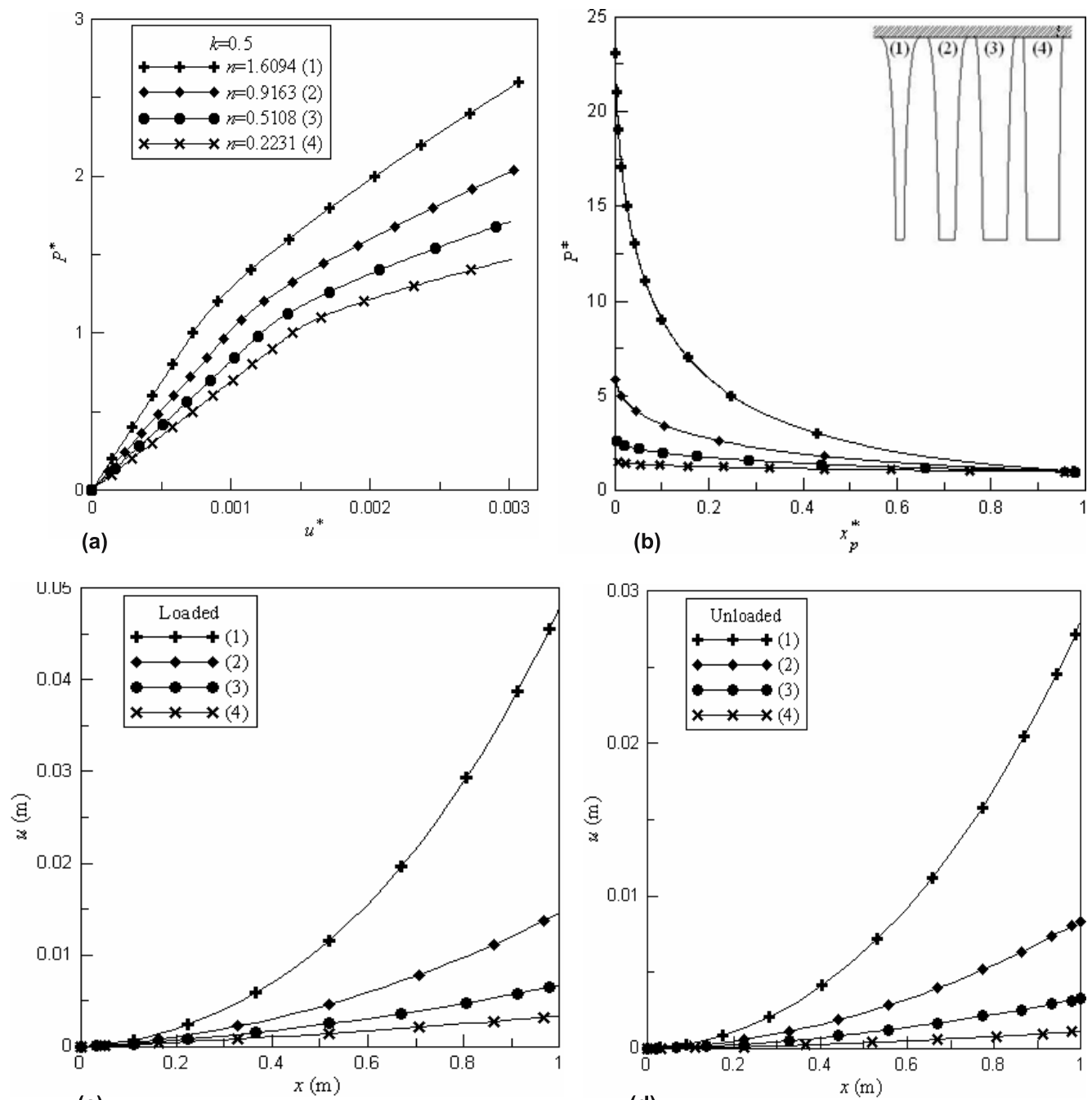

(c)

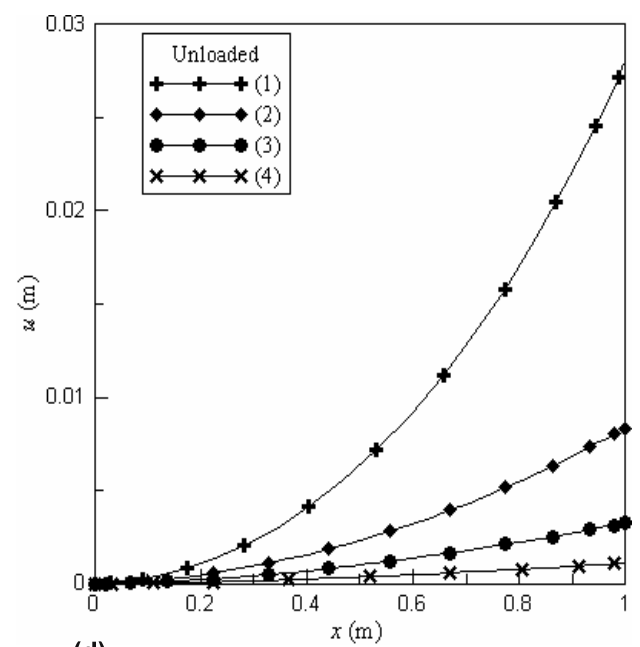

(d)

Figure 6. Plot for exponential bar under uniaxial tensile loading (a) $P^{*}$ vs $u^{*}$, (b) $P^{*}$ vs $x^{*}$, (c) $u$ vs $x$ corresponding to collapse loading and (d) $u$ vs $x$ after unloading.

figure 5 has also been followed here. In generating these results, the values of $n$ and $k$ are selected in such a way that the corresponding values of $d_{i}$ become identical to that used for figure 5 . The values of these two geometry controlling parameters have been indicated in the respective figures.

It is seen from figures 5-7 that the displacement plots (c) and corresponding residual displacement plots (d), are similar in nature. The slope of the displacement plots are conforming to the nature of strain corresponding to the geometry and external loading. Although, the collapse load depends on the fixed end diameter of the bar, the comparison of displacement and residual displacement plots for linearly taper, exponential and parabolic bars for same values of fixed and free end diameters reveals different amount of deformation both in loaded and unloaded condition. It is also observed that irrespective of the nature of taperness, be it lin- 

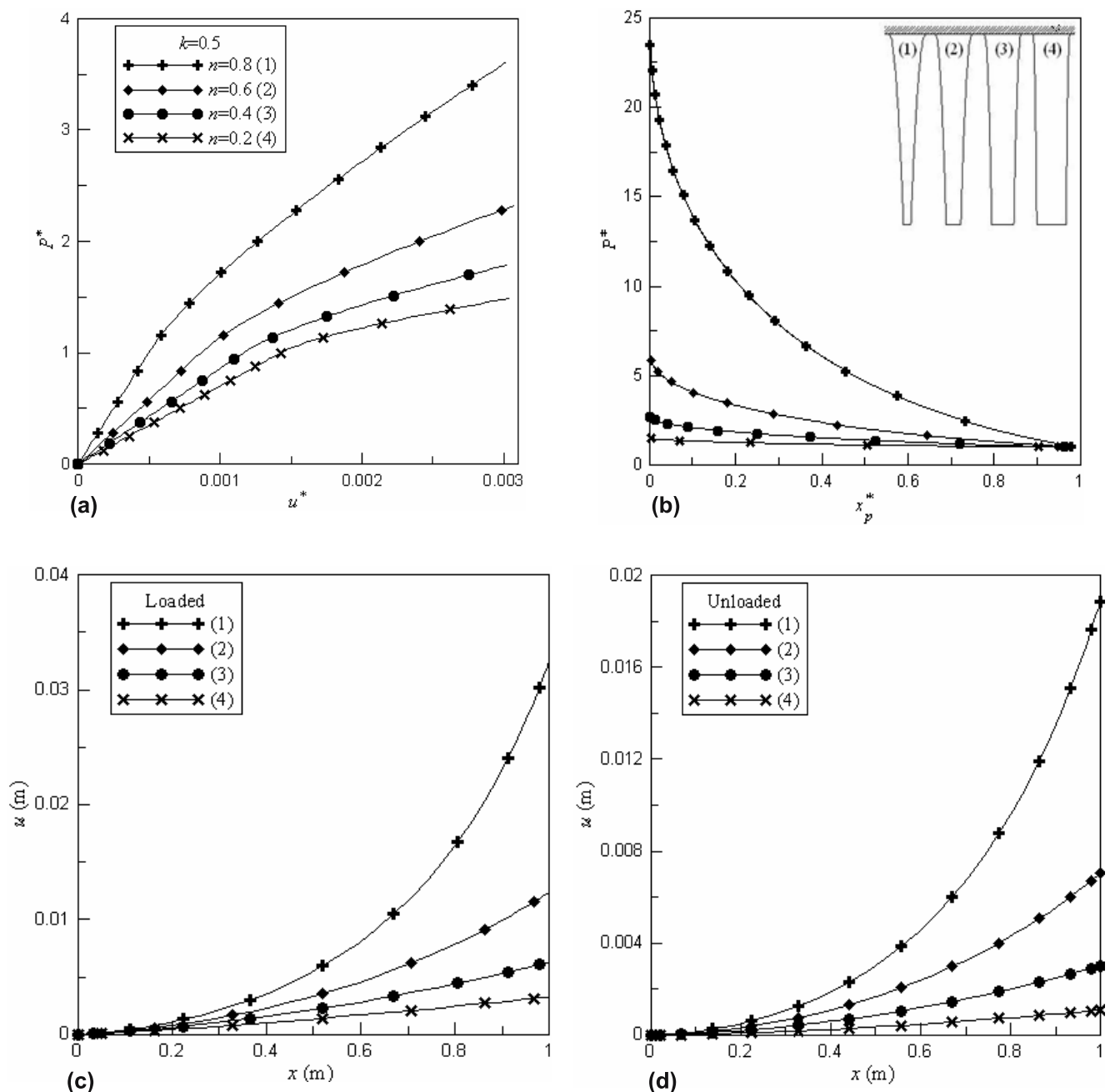

Figure 7. Plot for parabolic bar under uniaxial tensile loading (a) $P^{*}$ vs $u^{*}$, (b) $P^{*}$ vs $x^{*}$, (c) $u$ vs $x$ corresponding to collapse loading and (d) $u$ vs $x$ after unloading.

ear, exponential or parabolic, the deformation of the bar increases with decrease in free end diameter, which, is due to the fact that the elastic limit load decreases with reduction of free end diameter.

In all the plots shown in figures 5-7, the effect of material non-linearity is clearly seen from the normalized load-displacement plot. As the fixed end diameter of the bar is kept fixed at a constant value and the yield load increases with increase in the free end diameter, the resulting displacement is significantly reduced. For better understanding the yield limit loads $\left(P_{y}\right)$ and the collapse loads $\left(P_{c}\right)$ have been furnished in table 1(a). As the initiation of yielding occurs at free end, quite obviously the yield limit load is a function of tip diameter only and does not depend on types of geometry. At collapse load the elasto-plastic front reaches at the root and as the root diameter is same for all shapes and geometries, the value of collapse load is identical. However, the rate of advancement of plastic front with respect to the applied load is 
Table 1(a). Yield load $\left(P_{y}\right)$ and plastic collapse load $\left(P_{c}\right)$ for uniaxial tensile loading.

\begin{tabular}{lrc}
\hline$d_{i}(\mathrm{~m})$ & $P_{y}(\mathrm{~N})$ & $P_{c}(\mathrm{~N})$ \\
\hline 0.02 & 109955.74 & 2748893.57 \\
0.04 & 439822.97 & 2748893.57 \\
0.06 & 989601.69 & 2748893.57 \\
0.08 & 1759291.89 & 2748893.57 \\
\hline
\end{tabular}

Table 1(b). Permanent deformation $\left(\delta^{*}\right)$ for uniaxial tensile loading.

\begin{tabular}{lccc}
\hline & \multicolumn{3}{c}{$\delta^{*}\left(\times 10^{-3} \mathrm{~m}\right)$} \\
\cline { 2 - 4 }$d_{i}(\mathrm{~m})$ & Linear taper & Exponential & Parabolic \\
\hline 0.02 & 12.038 & 27.965 & 18.931 \\
0.04 & 4.810 & 8.363 & 7.034 \\
0.06 & 2.172 & 3.269 & 2.957 \\
0.08 & 0.082 & 1.122 & 1.086 \\
\hline
\end{tabular}

greatly accelerated with increase in the free end diameter, making it more sensitive to change in load as far as the movement of plastic front is concerned. Although the same trend is observed in case of all the profiles, the degree of plastic deformation and rate of plastic front movement with respect to the load vary by an appreciable amount. The amount of permanent deformation for all geometries considered here is given in table 1(b).

\subsection{Bar under body force loading}

The load-deflection behaviour for the linearly taper, exponential and parabolic bars are now considered under gravity loading with an additional exponential bar geometry $(k=1.5)$. The geometry and shape of the bars are kept same as in the case of uniform uniaxial loading, while an additional shape for exponential geometry has been considered. Once again plots similar to the ones for uniaxial tension loading have been shown in figures 8-11. However, unlike the case of tensile loading, here, the plots for displacement and residual displacement are presented in the same figure, namely part (c) of figures 8-11. The trends for $P^{*}$ vs $u^{*}$ and $P^{*}$ vs $x^{*}$ for a bar under increasing body force load remain the same as that of a bar under uniaxial tensile loading as far as the effect of reducing the free end diameter is concerned. But the significant observation in this case is that the position of yielding initiation can be anywhere in between the free and fixed end sections (but certainly not at the free end as it is a stress free surface) and accordingly the plastic front may proceed in one direction or in both the directions depending on the position of the initiation of yielding. Also it can be found from any of these plots that the rate of advancement of plastic front with respect to body force differs by a very small amount from each other.

It is seen from part (c) of figures 8-11 that the slope of the displacement of the bar conforms to the nature of loading with a value zero at the free surface. Like the earlier case of tensile loading, the residual displacement field is obtained through an elastic unloading from the collapse load following linear elastic material behaviour with elastic modulus $E$. 


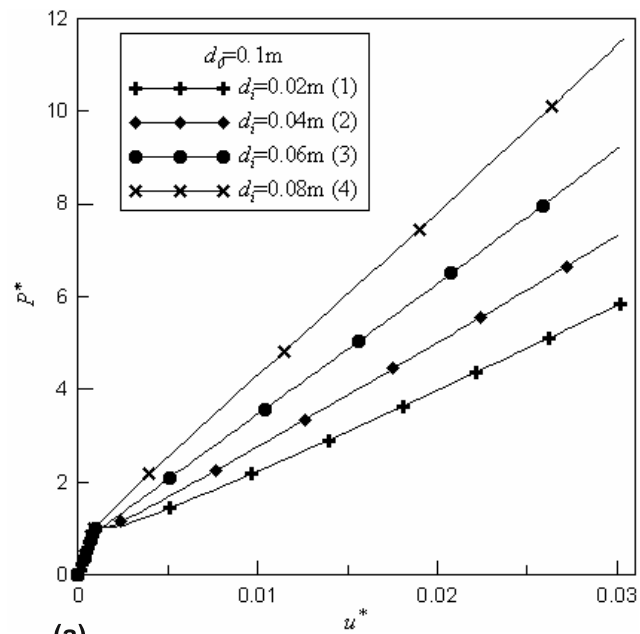

(a)



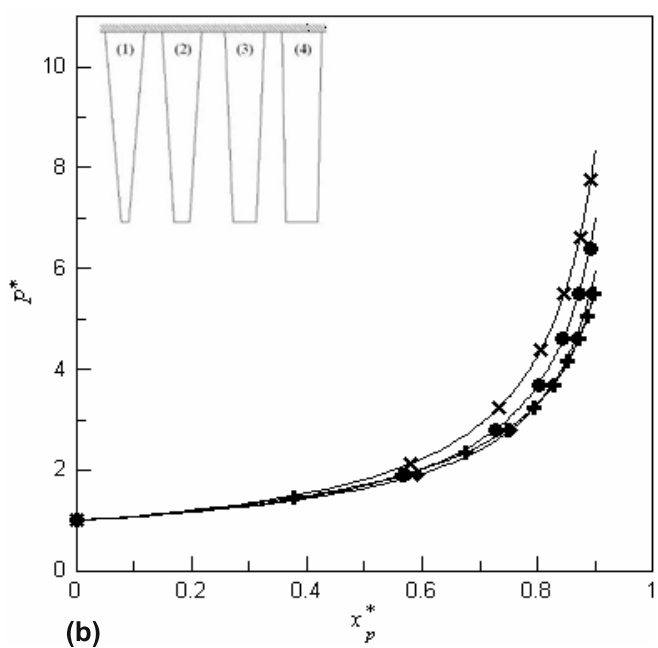

(b)

Figure 8. Plot for linearly taper bar under body force loading (a) $P^{*}$ vs $u^{*}$, (b) $P^{*}$ vs $x^{*}$ and (c) $u$ vs $x$ for loading corresponding to $x_{p}^{*}=0.9$ and after unloading.

For body force type of loading initial yielding does not occur at the free end and hence depending on the geometry of the bar the intensity of yield limit gravity loading and the location of yielding are different, even when the tip diameter remains same. The numerical values of plastic limit load and location of elasto-plastic front corresponding to initial yielding have been furnished in table 2(a) for all the geometries whereas in table 2(b) the amount of permanent deformation is furnished when $90 \%$ of the bar is under post-elastic regime. As the free end is stress free surface, theoretically no load would be sufficient to produce plastic collapse of the bar. The results indicate intermediate location of the initiation of yielding for two particular cases (figures 9 and 11), where the plastic front grows in both directions. With increasing load, one end of the plastic front coalesces with fixed end, and to get more detailed information figures 9(b) and 11(b) are plotted once again in figure 12, with semi log axes. The table and the plots indicate existence of an optimized profile and geometry of the bar under different type of loading depending on the requirements of the user. 




(a)

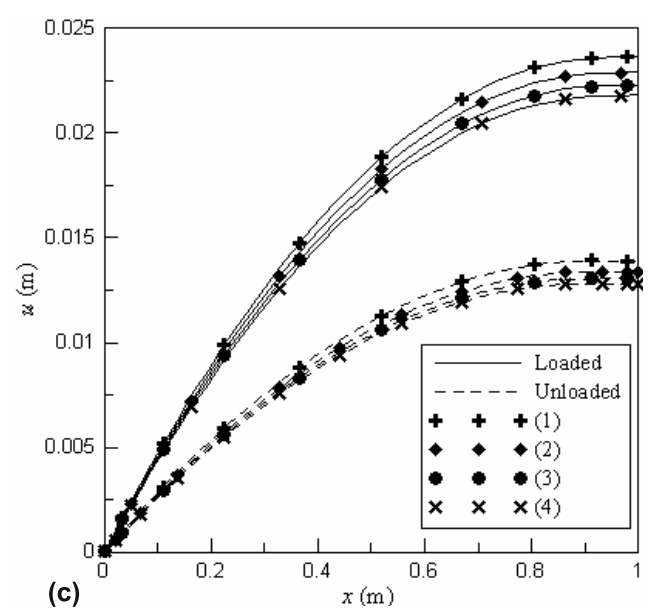



(b)

Figure 9. Plot for exponential bar under body force loading (a) $P^{*}$ vs $u^{*}$, (b) $P^{*}$ vs $x^{*}$ and (c) $u$ vs $x$ for loading corresponding to $x_{p}^{*}=0.9$ and after unloading.

The present formulation is based on non-linear strain-displacement relation. The problems dealt in the present paper can also be formulated with linear strain-displacement relation $\left(\varepsilon_{x}=\frac{d u}{d x}\right)$. Normalized load-displacement plots for linearly taper, exponential and parabolic bar under tensile loading have been generated using linear strain-displacement relation for $d_{0}=0.1 \mathrm{~m}$ and $d_{i}=0.02 \mathrm{~m}$ and presented in figure 13. Results for similar geometries and loading using non-linear strain-displacement relation have also been presented in the same figure. The plot shows insignificant difference in the results obtained using linear and nonlinear strain displacement relation, indicating a negligible effect of geometric non-linearity in comparison to material non-linearity.

\section{Post-elastic vibration of taper bar}

In order to establish the robustness of the present method a dynamic problem is taken up. The problem is to investigate the elastic and post-elastic dynamic behaviour of solid circular 

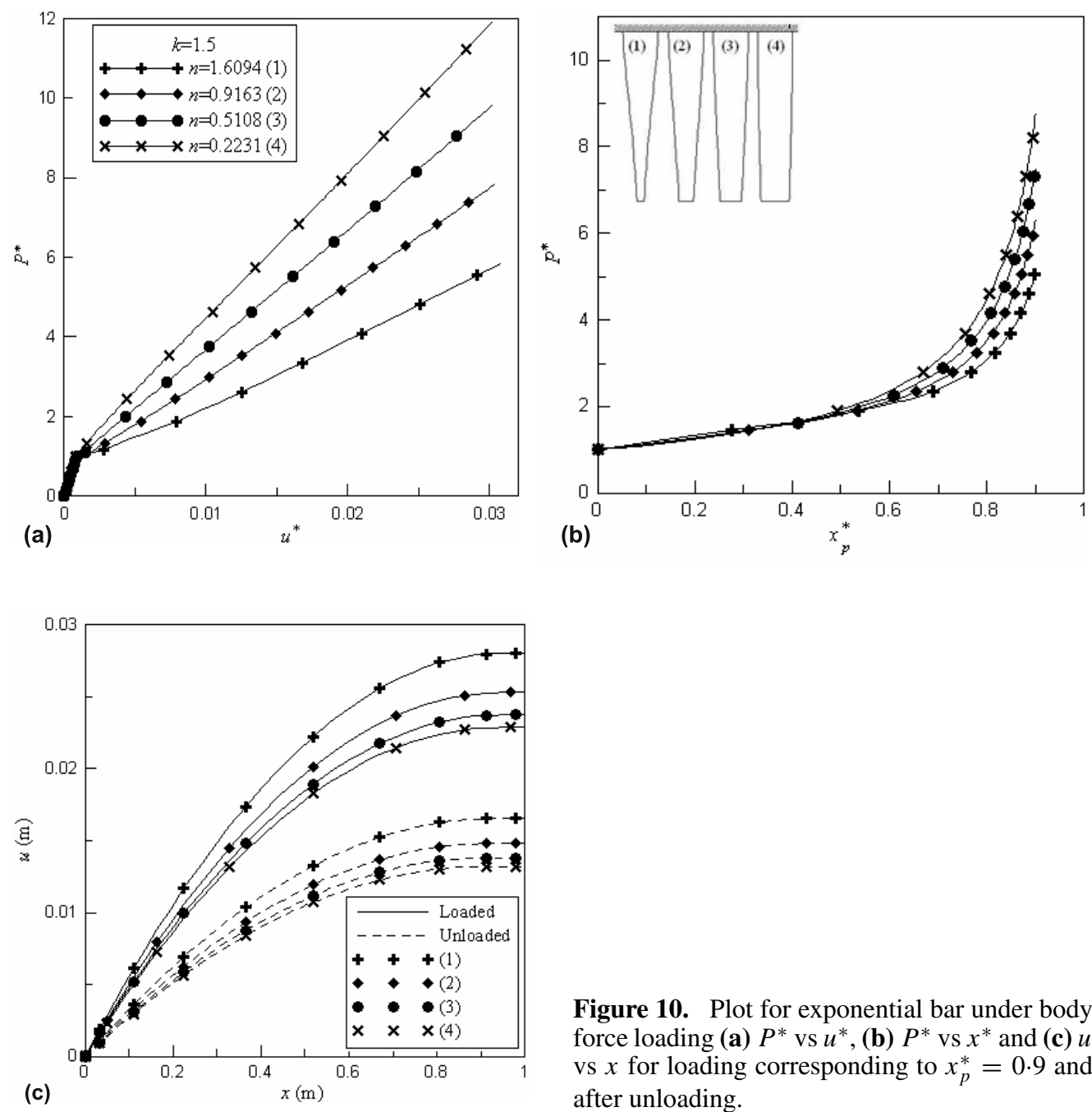

Figure 10. Plot for exponential bar under body force loading (a) $P^{*}$ vs $u^{*}$, (b) $P^{*}$ vs $x^{*}$ and (c) $u$ vs $x$ for loading corresponding to $x_{p}^{*}=0.9$ and after unloading.

non-uniform taper bar in terms of loaded natural frequency. To the best knowledge of the authors, no work is available in the literature for prediction of post-elastic dynamic behaviour of taper bar. This problem has significant engineering importance in order to make the design competitive.

As the aim is to calculate the transverse natural frequencies of bar in loaded condition, transverse displacement $w$ is also taken into mathematical formulation in addition to the inplane displacement $u$. The formulation is based in two parts. First, the static problem of a uniaxially loaded bar is solved using the same formulation as mentioned in sections $2 \cdot 1-2 \cdot 2$. In the next step, the dynamic problem is formulated using the unknown parameters $c_{i}$ that are obtained from the static problem. As the static formulation is already mentioned in sections $2 \cdot 1-2 \cdot 2$, it is not repeated to maintain brevity. This methodology of dynamic problem has been adopted in Saha et al (2005) for analysis of non-linear vibration of rectangular plates in the elastic region. 

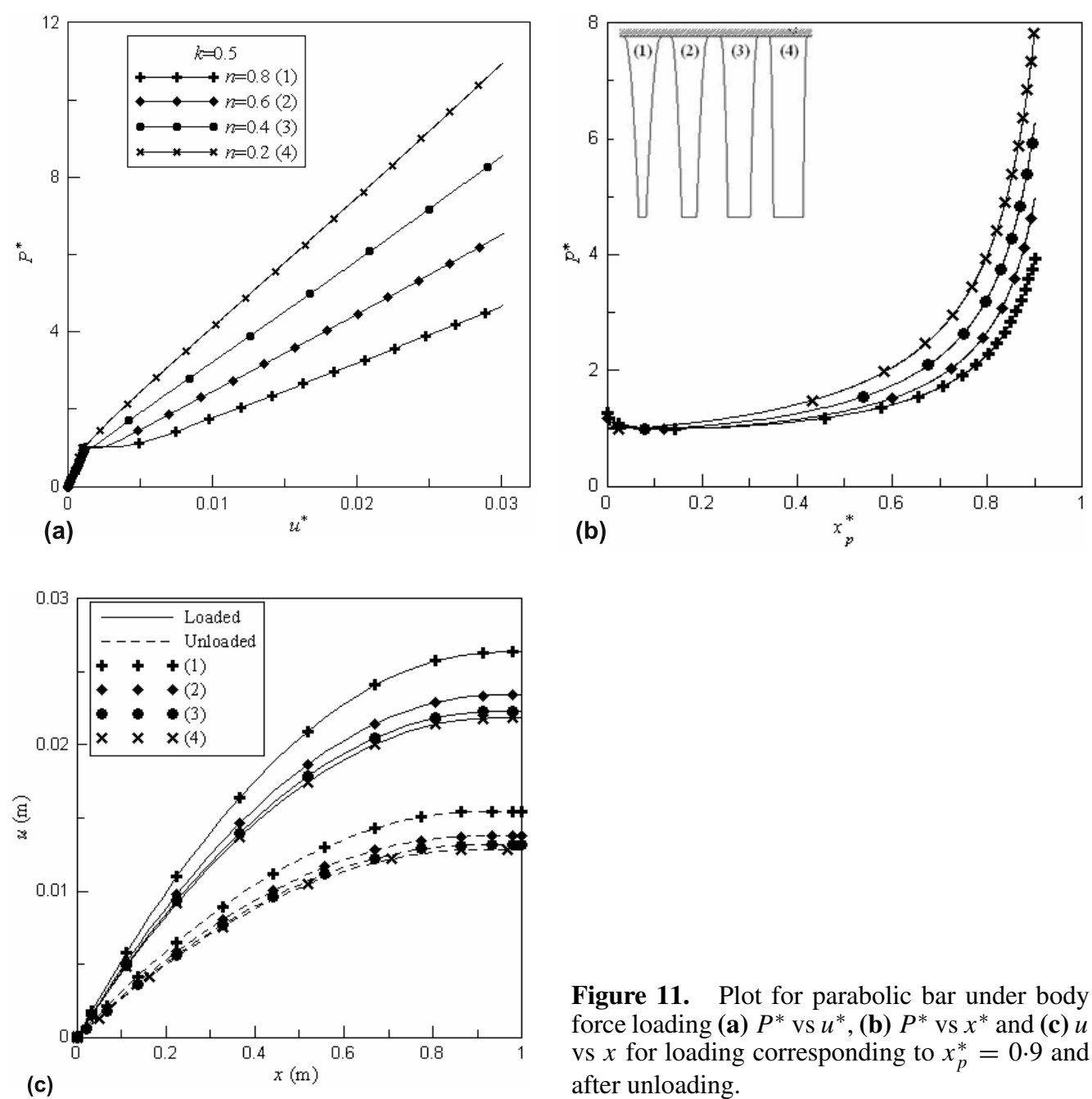

Figure 11. Plot for parabolic bar under body force loading (a) $P^{*}$ vs $u^{*}$, (b) $P^{*}$ vs $x^{*}$ and (c) $u$ vs $x$ for loading corresponding to $x_{p}^{*}=0.9$ and after unloading.

In the present formulation, the strain energy for bending and stretching due to $w$ has been considered for the reason stated earlier. Also, in this formulation, the non-linear part (second order) in strain displacement expression of $u$ is not taken into account due to its negligible contribution in the static displacement as shown in figure 13. The expression of strain at any fibre at a distance $y$ from the neutral axis due to combined effect of $u$ and $w$ is given by,

$$
\varepsilon=-y \frac{\partial^{2} w}{\partial x^{2}}+\frac{1}{2}\left(\frac{\partial w}{\partial x}\right)^{2}+\frac{\partial u}{\partial x}
$$

The elastic and post-elastic part of strain energies are given by,

$$
U_{e}=\frac{\pi E}{8} \int_{0}^{L_{e}}\left\{\frac{d^{4}}{16}\left(\frac{\partial^{2} w}{\partial x^{2}}\right)^{2}+\frac{d^{2}}{4}\left(\frac{\partial w}{\partial x}\right)^{4}+d^{2}\left(\frac{\partial u}{\partial x}\right)^{2}+d^{2}\left(\frac{\partial w}{\partial x}\right)^{2} \frac{\partial u}{\partial x}\right\} d x
$$




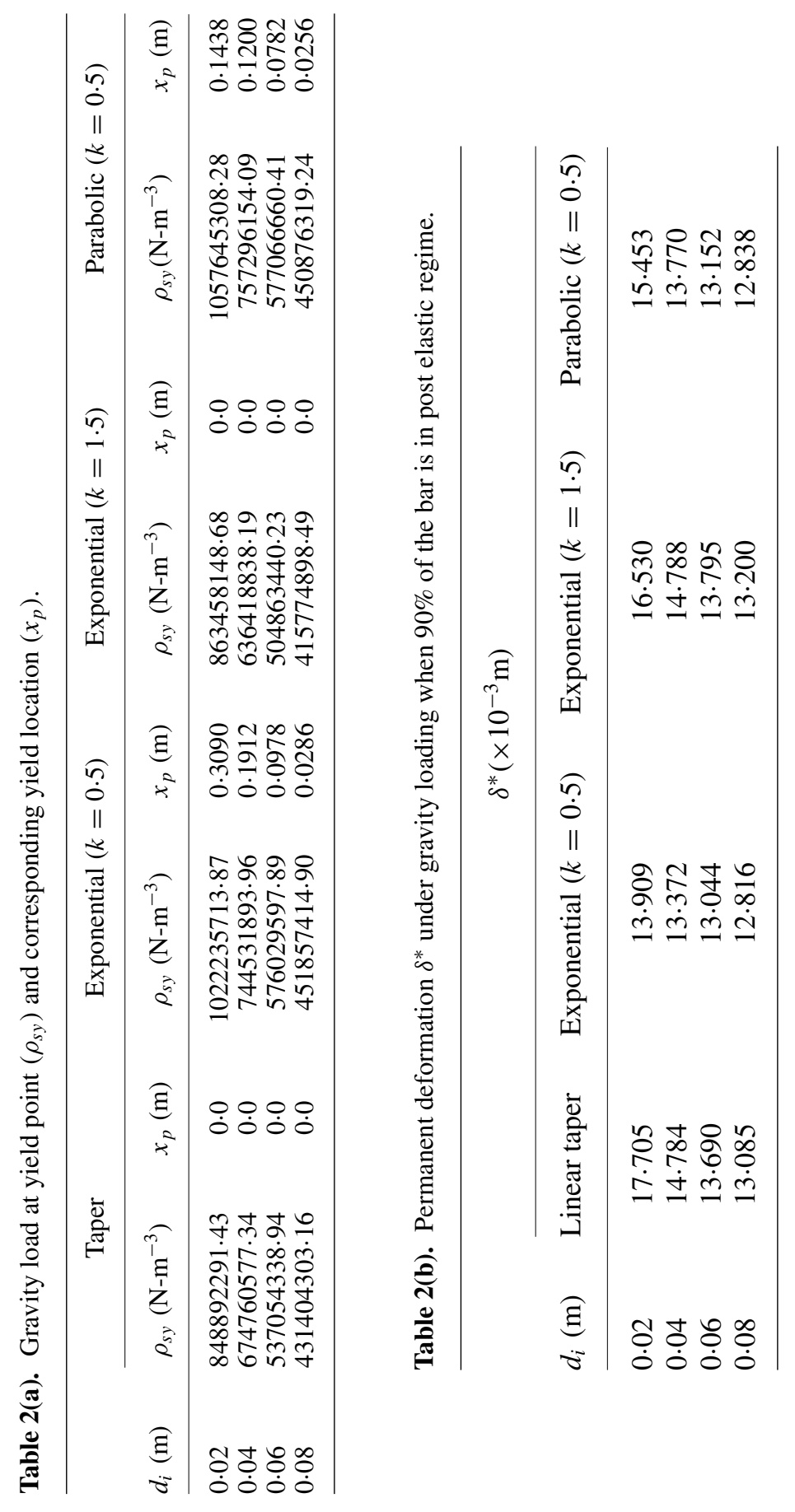




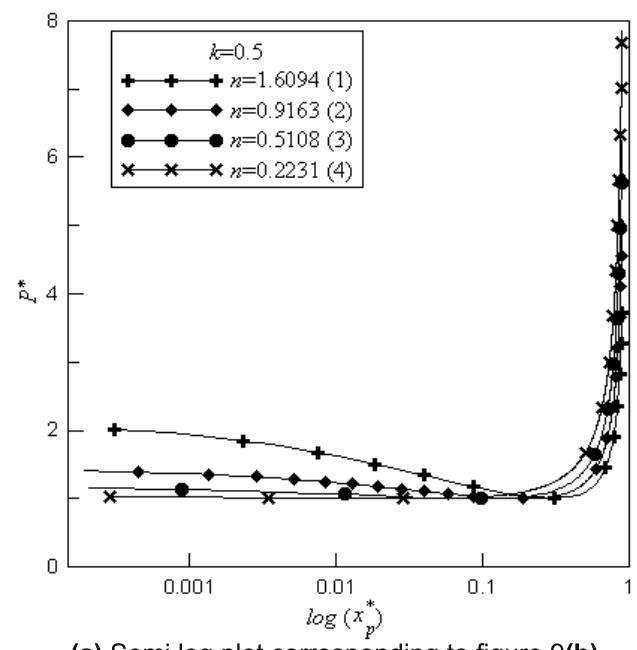

(a) Semi log plot corresponding to figure 9 (b)



(b) Semi log plot corresponding to figure 11(b)

Figure 12. Semi log plot of yield locations corresponding to figure $9(\mathbf{b})$ and $11(\mathbf{b})$.

and

$$
\begin{aligned}
U_{p}= & \frac{\pi E_{1}}{8} \int_{L_{e}}^{L}\left\{\frac{d^{4}}{16}\left(\frac{\partial^{2} w}{\partial x^{2}}\right)^{2}+\frac{d^{2}}{4} A(x)\left(\frac{\partial w}{\partial x}\right)^{4}+d^{2}\left(\frac{\partial u}{\partial x}\right)^{2}+d^{2}\left(\frac{\partial w}{\partial x}\right)^{2} \frac{\partial u}{\partial x}\right\} d x \\
& +\frac{\pi\left(E-E_{1}\right)}{4} \varepsilon_{y} \int_{L_{e}}^{L} d^{2}\left\{\frac{1}{2}\left(\frac{\partial w}{\partial x}\right)^{2}+\frac{\partial u}{\partial x}\right\} d x-\frac{\pi\left(E-E_{1}\right)}{8} \varepsilon_{y}^{2} \int_{L_{e}}^{L} d^{2} d x
\end{aligned}
$$

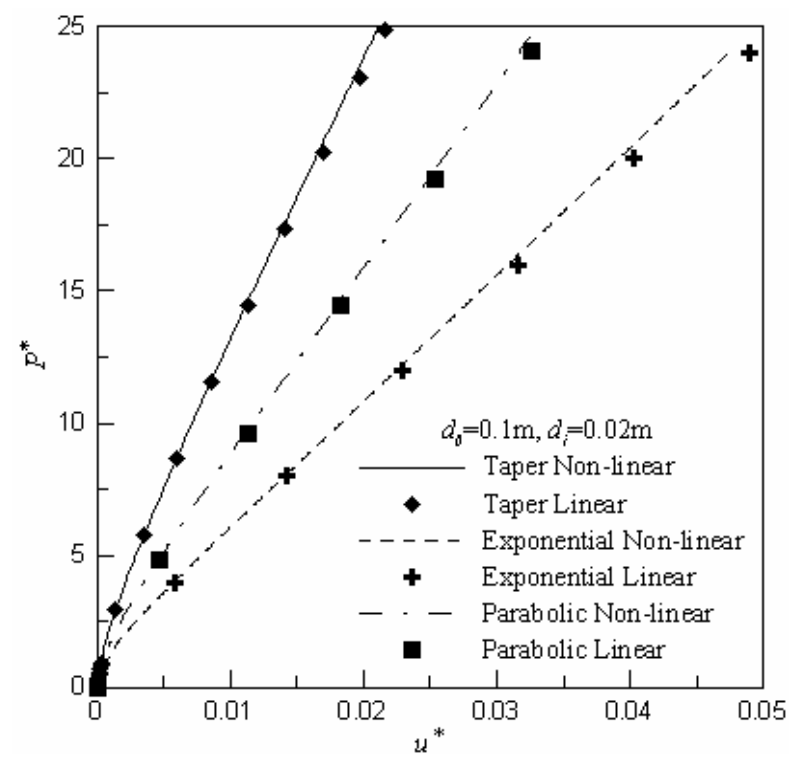

Figure 13. Comparison plot between linear and non-linear straindisplacement formulation under tensile loading. 


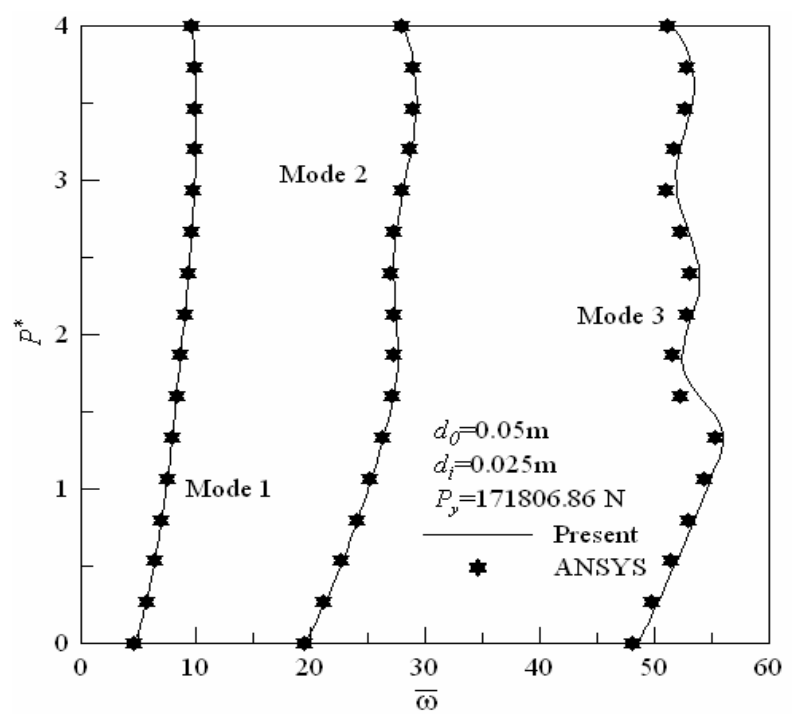

Figure 14. Variation of nondimensional free vibration frequency of linearly taper bar with normalized axial load for first three modes.

The expressions in (18) and (19) are obtained using the same method as mentioned for static analysis, i.e. calculating the area under stress strain diagram using the expression in (17).

The variational form of the dynamic problem is obtained from Hamilton's principle which states that

$$
\delta\left(\int_{\tau_{1}}^{\tau_{2}} L^{\prime} d \tau\right)=0
$$

where $L^{\prime}=T-(U+V)$ is called the Lagrangian and $T, U$ and $V$ are the total kinetic energy, strain energy and potential energy of the external forces respectively. It should be noted that the value of $V$ is taken as zero as it is a case of free vibration.

The expression of kinetic energy $T$ is given by,

$$
T=\frac{\pi}{8} \rho \int_{0}^{L} d^{2}\left\{\left(\frac{\partial w}{\partial \tau}\right)^{2}+\left(\frac{\partial u}{\partial \tau}\right)^{2}\right\} d x,
$$

where $\rho$ is the density of the bar material and $\tau$ represents time.

The dynamic displacements $w(\xi, \tau)$ and $u(\xi, \tau)$ are assumed to be separable in space and time as shown below:

$$
\begin{aligned}
& w(\xi, \tau)=\sum_{i=1}^{n f^{\prime}} c_{i} \alpha_{i}(\xi) \gamma_{i}(\tau) \\
& u(\xi, \tau)=\sum_{i=n f^{\prime}+1}^{n f^{\prime}+n f} c_{i} \phi_{i-n f^{\prime}}(\xi) \gamma_{i-n f^{\prime}}(\tau) .
\end{aligned}
$$

Here, $n f^{\prime}$ denotes the number of functions for $w$ and $c_{i}$ is a new set of unknown parameters to be evaluated, which indicates the contribution of the individual vibration modes for a particular vibration frequency. The space function $u$ is completely known from the static 
analysis. It must be mentioned that the unknown coefficients corresponding to transverse displacement are taken as zero as the bar retains its initial straight configuration due to axial uniform loading. The set of temporal functions is expressed by $\gamma_{i}=e^{i \omega \tau}$, where, $\omega$ represents the natural frequency of the system and $\mathrm{i}=\sqrt{-1}$.

Putting equations (18), (19) and (21) in equation (20) and using expressions of the dynamic displacement fields from equation (22) the governing equations of the dynamic problem is obtained in the following form,

$$
-\omega^{2}[M]\{c\}+[K]\{c\}=0 .
$$

Here, $[K]$ and $[M]$ are stiffness matrix and mass matrix which are of the form given below,

$$
[K]=\left[\begin{array}{cc}
{\left[K_{11}\right]} & {\left[K_{12}\right]} \\
{\left[K_{22}\right]} & {\left[K_{22}\right]}
\end{array}\right] \text { and }[M]=\left[\begin{array}{cc}
{\left[M_{11}\right]} & 0 \\
0 & {\left[M_{22}\right]}
\end{array}\right] .
$$

The elements of stiffness matrix and mass matrix are provided below:

$$
\begin{aligned}
& {\left[K_{11}\right]=\frac{\pi E}{64 L_{e}^{3}} \sum_{j=1}^{n f^{\prime}} \sum_{i=1}^{n f^{\prime}} \int_{0}^{1} d^{4}\left(\frac{\partial^{2} \alpha_{i}^{e}}{\partial \xi_{1}^{2}}\right)\left(\frac{\partial^{2} \alpha_{j}^{e}}{\partial \xi_{1}^{2}}\right) d \xi_{1}} \\
& +\frac{\pi E}{8 L_{e}^{3}} \sum_{j=1}^{n f^{\prime}} \sum_{i=1}^{n f^{\prime}} \int_{0}^{1} d^{2}\left(\sum_{i=1}^{n f^{\prime}} c_{i} \frac{\partial \alpha_{i}^{e}}{\partial \xi_{1}}\right)^{2}\left(\frac{\partial \alpha_{i}^{e}}{\partial \xi_{1}}\right)\left(\frac{\partial \alpha_{j}^{e}}{\partial \xi_{1}}\right) d \xi_{1} \\
& +\frac{\pi E}{4 L_{e}^{2}} \sum_{j=1}^{n f^{\prime}} \sum_{i=1}^{n f^{\prime}} \int_{0}^{1} d^{2}\left(\sum_{i=n f^{\prime}+1}^{n f^{\prime}+n f} c_{i} \frac{\partial \phi_{i-n f^{\prime}}^{e}}{\partial \xi_{1}}\right)\left(\frac{\partial \alpha_{i}^{e}}{\partial \xi_{1}}\right)\left(\frac{\partial \alpha_{j}^{e}}{\partial \xi_{1}}\right) d \xi_{1} \\
& +\frac{\pi E_{1}}{64 L_{p}^{3}} \sum_{j=1}^{n f^{\prime}} \sum_{i=1}^{n f^{\prime}} \int_{0}^{1} d^{4}\left(\frac{\partial^{2} \alpha_{i}^{p}}{\partial \xi_{2}^{2}}\right)\left(\frac{\partial^{2} \alpha_{j}^{p}}{\partial \xi_{2}^{2}}\right) d \xi_{2} \\
& +\frac{\pi E_{1}}{8 L_{p}^{3}} \sum_{j=1}^{n f^{\prime}} \sum_{i=1}^{n f^{\prime}} \int_{0}^{1} d^{2}\left(\sum_{i=1}^{n f^{\prime}} c_{i} \frac{\partial \alpha_{i}^{p}}{\partial \xi_{2}}\right)^{2}\left(\frac{\partial \alpha_{i}^{p}}{\partial \xi_{2}}\right)\left(\frac{\partial \alpha_{j}^{p}}{\partial \xi_{2}}\right) d \xi_{2} \\
& +\frac{\pi E_{1}}{4 L_{p}^{2}} \sum_{j=1}^{n f^{\prime}} \sum_{i=1}^{n f^{\prime}} \int_{0}^{1} d^{2}\left(\sum_{i=n f^{\prime}+1}^{n f^{\prime}+n f} c_{i} \frac{\partial \phi_{i-n f^{\prime}}^{p}}{\partial \xi_{2}}\right)\left(\frac{\partial \alpha_{i}^{p}}{\partial \xi_{2}}\right)\left(\frac{\partial \alpha_{j}^{p}}{\partial \xi_{2}}\right) d \xi_{2} \\
& +\frac{\pi\left(E-E_{1}\right)}{4 L_{p}} \varepsilon_{y} \sum_{j=1}^{n f^{\prime}} \sum_{i=1}^{n f^{\prime}} \int_{0}^{1} d^{2}\left(\frac{\partial \alpha_{i}^{p}}{\partial \xi_{2}}\right)\left(\frac{\partial \alpha_{j}^{p}}{\partial \xi_{2}}\right) d \xi_{2}, \quad\left[K_{12}\right]=0 \\
& {\left[K_{21}\right]=\frac{\pi E}{8 L_{e}^{2}} \sum_{j=n f^{\prime}+1}^{n f^{\prime}+n f} \sum_{i=1}^{n f^{\prime}} \int_{0}^{1} d^{2}\left(\sum_{i=1}^{n f^{\prime}} c_{i} \frac{\partial \alpha_{i}^{e}}{\partial \xi_{1}}\right)\left(\frac{\partial \alpha_{i}^{e}}{\partial \xi_{1}}\right)\left(\frac{\partial \phi_{j-n f^{\prime}}^{e}}{\partial \xi_{1}}\right) d \xi_{1},} \\
& +\frac{\pi E_{1}}{8 L_{p}^{2}} \sum_{j=n f^{\prime}+1}^{n f^{\prime}+n f} \sum_{i=1}^{n f^{\prime}} \int_{0}^{1} d^{2}\left(\sum_{i=1}^{n f^{\prime}} c_{i} \frac{\partial \alpha_{i}^{p}}{\partial \xi_{2}}\right)\left(\frac{\partial \alpha_{i}^{p}}{\partial \xi_{2}}\right)\left(\frac{\partial \phi_{j-n f^{\prime}}^{p}}{\partial \xi_{2}}\right) d \xi_{2},
\end{aligned}
$$




$$
\begin{aligned}
{\left[K_{22}\right]=} & \frac{\pi E}{4 L_{e}} \sum_{j=n f^{\prime}+1}^{n f^{\prime}+n f} \sum_{i=n f^{\prime}+1}^{n f^{\prime}+n f} \int_{0}^{1} d^{2}\left(\frac{\partial \phi_{i-n f^{\prime}}^{e}}{\partial \xi_{1}}\right)\left(\frac{\partial \phi_{j-n f^{\prime}}^{e}}{\partial \xi_{1}}\right) d \xi_{1} \\
& +\frac{\pi E_{1}}{4 L_{p}} \sum_{j=n f^{\prime}+1}^{n f^{\prime}+n f} \sum_{i=n f^{\prime}+1}^{n f^{\prime}+n f} \int_{0}^{1} d^{2}\left(\frac{\partial \phi_{i-n f^{\prime}}^{p}}{\partial \xi_{2}}\right)\left(\frac{\partial \phi_{j-n f^{\prime}}^{p}}{\partial \xi_{2}}\right) d \xi_{2}, \\
{\left[M_{11}\right]=} & \frac{\pi L \rho}{4} \sum_{j=1}^{n f^{\prime}} \sum_{i=1}^{n f^{\prime}} \int_{0}^{1} d^{2} \alpha_{i} \alpha_{j} d \xi, \\
{\left[M_{22}\right]=} & \frac{\pi L \rho}{4} \sum_{j=n f^{\prime}+1}^{n f^{\prime}+n f} \sum_{i=n f^{\prime}+1}^{n f^{\prime}+n f} \int_{0}^{1} L \rho \phi_{i-n f^{\prime}} \phi_{j-n f^{\prime}} d \xi .
\end{aligned}
$$

Functions for both $w$ and $u$ are broken into two parts, one corresponding to elastic region and the other one corresponding to post-elastic region. Non-linear terms of the stiffness matrix are linearized by using unknown coefficients obtained from the static solution.

Equation (23) can be transformed into $\left[M^{-1} K\right]\{c\}-\omega^{2}\{c\}=0$, which is a standard eigen value problem. The eigen values are solved numerically by using IMSL routines and the square roots of these eigen values represent the loaded free vibration frequencies of the bar. The transverse displacement field corresponding to each of the eigen values gives the mode shapes of loaded vibration frequencies.

\subsection{Validation of the dynamic problem}

The variation of free vibration frequency of first three modes of linearly taper bar with axial load has been presented in figure 14 in non-dimensional load-frequency plane and this result is contrasted with that obtained using ANSYS (version 8.0). Non-dimensional frequency $\bar{\omega}$ is given by $\bar{\omega}=\omega L^{2} \sqrt{\rho / E I_{0}}$, where $\omega$ is the frequency of vibration in $\mathrm{rad} / \mathrm{s}$ and $I_{0}$ is the area moment of inertia of the bar cross section at the fixed end. Results are generated using $E=210 \mathrm{GPa}, E_{1}=70 \mathrm{GPa}, \sigma_{y}=350 \mathrm{MPa}, \rho=7850 \mathrm{Kg} / \mathrm{m}^{3}, L=1 \mathrm{~m}, d_{0}=0.05 \mathrm{~m}$ and $d_{i}=0.025 \mathrm{~m}$. The pseudo-code for the problem in ANSYS is written using pre-stressed modal analysis procedures and the bar is modelled using BEAM188 with 50 elements. The validation shows good agreement. Also, the mode shapes for the first three modes of the same problem have been shown in figure 15. The mode shapes are shown for two different loads, one at yield load $P_{y}\left(P^{*}=1\right)$ and the other at the collapse load $P_{c}\left(P^{*}=4\right)$. The comparison of corresponding mode shapes clearly shows the difference in the dynamic behaviour at elastic and post-elastic condition.

\section{Two-dimensional problem}

The present method of analysis has been extended for a sample two-dimensional problem, in which elasto-plastic analysis of a uniform rotating solid disk with radius $b$ under centrifugal body force loading with plane stress assumption has been carried out. Only a brief description of the formulation and validation on plastic limit angular speed has been presented in this paper to maintain brevity. 


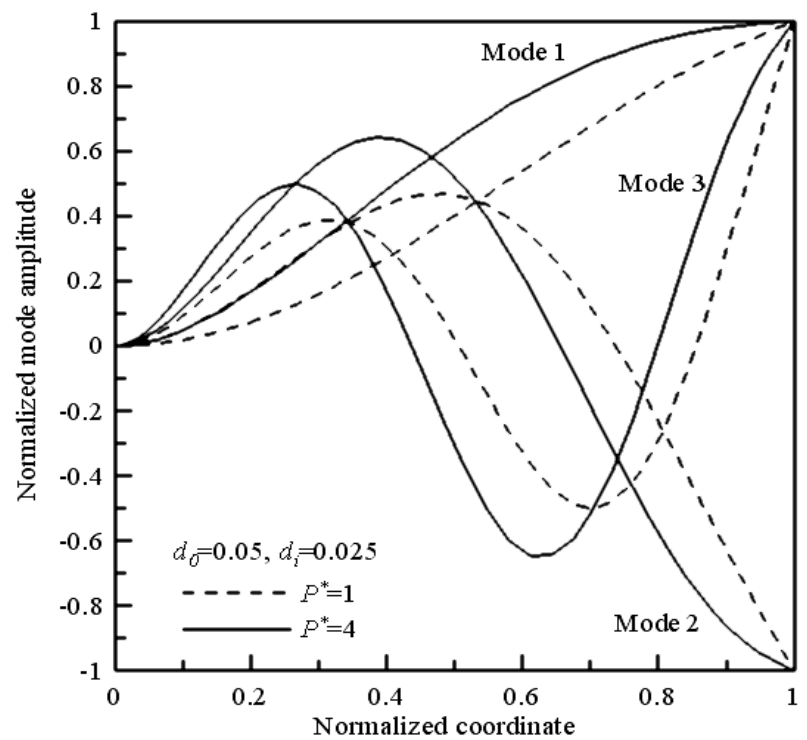

Figure 15. Mode shape plot at yield load and plastic collapse load for the linearly taper bar of figure 14 .

Due to rotational symmetry of geometry, loading and boundary condition, an axisymmetric analysis has been carried out. With the presence of centrifugal loading induced by the rotation of the disk, radial and tangential stresses will be induced in the disk which are principle stresses in this case. The strain energy for elastic and post-elastic part of disk have been calculated by considering the area under both radial and tangential stress-strain diagram. It is to be noted that von-Mises yield criterion has been used to predict the onset of yielding. For a rotating solid disk, yielding initiates at the centre of the disk and the corresponding angular speed is termed as elastic limit angular speed. With gradual increase in rotational speed, plastic front (point of initiation of yielding) proceeds towards the outer radius. When the plastic front reaches the outer radius, i.e. when the whole disk just reaches the plastic state, the corresponding angular speed is termed as plastic limit angular speed.

Expression of the elastic part of strain energy for the outer region is given by,

$$
\begin{aligned}
U_{e} & =\frac{1}{2} \int_{V o l}(\sigma \varepsilon) d v=\frac{1}{2} \int_{V o l}\left(\sigma_{t} \varepsilon_{t}+\sigma_{r} \varepsilon_{r}\right) d v \\
& =\frac{\pi E}{1-\mu^{2}} \int_{r_{c}}^{b}\left\{\frac{u^{2}}{r}+2 \mu u\left(\frac{d u}{d \mathrm{r}}\right)+r\left(\frac{d u}{d r}\right)^{2}\right\} h d r,
\end{aligned}
$$

where the subscript $r$ and $t$ denote radial and tangential direction, $\mu$ is the Poisson's ratio, $h$ is disk thickness and $r_{c}$ denotes the location of plastic front at any intermediate speed in between elastic and plastic limit angular speed. A pictorial representation of the disk has been depicted in figure 16 corresponding to this state of stress. In arriving at equation (24), the expressions used for radial and tangential strains and stresses are given by,

$$
\begin{aligned}
\varepsilon_{r} & =\frac{d u}{d r} \text { and } \varepsilon_{t}=\frac{u}{r} ; \sigma_{r}=\frac{E}{\left(1-\mu^{2}\right)}\left(\varepsilon_{r}+\mu \varepsilon_{t}\right) \text { and } \sigma_{t} \\
& =\frac{E}{\left(1-\mu^{2}\right)}\left(\varepsilon_{t}+\mu \varepsilon_{r}\right) .
\end{aligned}
$$



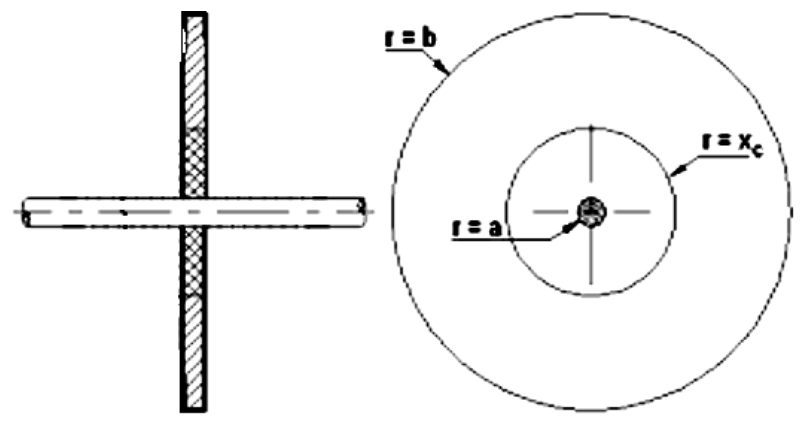

Figure 16. Rotating disk under elasto-plastic state of stress.

The post-elastic part of strain energy is given by,

$$
\begin{aligned}
U_{p}= & \frac{1}{2} \frac{E_{1}}{\left(1-\mu^{2}\right)} \int_{0}^{r_{c}}\left\{\frac{u^{2}}{r}+r\left(\frac{d u}{d r}\right)^{2}+2 \mu u \frac{d u}{d r}\right\} 2 \pi h d r \\
& +\frac{E-E_{1}}{\left(1-\mu^{2}\right)} \int_{0}^{r_{c}}\left\{\left(\varepsilon_{r}^{0}+\mu \varepsilon_{t}^{0}\right) r \frac{d u}{d r}+\left(\varepsilon_{t}^{0}+\mu \varepsilon_{r}^{0}\right) u\right\} 2 \pi h d r \\
& -\frac{E-E_{1}}{2\left(1-\mu^{2}\right)} \int_{0}^{r_{c}}\left\{\left(\varepsilon_{r}^{0}\right)^{2} r+\left(\varepsilon_{t}^{0}\right)^{2} r+2 \mu \varepsilon_{t}^{0} \varepsilon_{r}^{0} r\right\} 2 \pi h d r .
\end{aligned}
$$

In deriving equation (26), the expressions for $\overline{\varepsilon_{r}}$ and $\overline{\varepsilon_{t}}$ are decomposed as $\varepsilon_{r}=\varepsilon_{r}^{p}+\varepsilon_{r}^{0}$ and $\varepsilon_{t}=\varepsilon_{t}^{p}+\varepsilon_{t}^{0}$ whereas, the expressions used for the decomposed components of $\overline{\sigma_{r}}$ and $\overline{\sigma_{t}}$ are given by: $\sigma_{r}^{0}=\frac{E}{\left(1-\mu^{2}\right)}\left[\varepsilon_{r}^{0}+v \varepsilon_{t}^{0}\right], \sigma_{r}^{p}=\frac{E_{1}}{\left(1-\mu^{2}\right)}\left[\varepsilon_{r}^{p}+v \varepsilon_{t}^{p}\right], \sigma_{t}^{0}=\frac{E}{\left(1-\mu^{2}\right)}\left[\varepsilon_{t}^{0}+v \varepsilon_{r}^{0}\right]$ and $\sigma_{t}^{p}=\frac{E_{1}}{\left(1-\mu^{2}\right)}\left[\varepsilon_{t}^{p}+v \varepsilon_{r}^{p}\right]$. It is to be noted that the numerical values of $\sigma_{r}^{0}$ and $\sigma_{t}^{0}$ are not identical with the yield stress value $\sigma_{y}$ in general, but the equivalent von-Mises stress $\sigma_{0}$ obtained from $\sigma_{r}^{0}$ and $\sigma_{t}^{0}$ attains the yield stress value.

The expression for work potential is given below, in which the rotational speed $\omega$ acts as the forcing function.

$$
V=-\int_{v o l} u\left(\omega^{2} r\right) d m-2 \pi \rho \omega^{2} \int_{0}^{b}\left(r^{2} u h\right) d r,
$$

where $\rho$ is the density of the disk material. Considering the global displacement field as the linear combination of unknown coefficient and orthogonal admissible functions and dividing the global displacement field into elastic and post-elastic part, the extension of minimum potential energy principle yields the following governing equation

$$
\begin{aligned}
& \frac{E}{1-\mu^{2}} \sum_{j=1}^{n f} \sum_{i=1}^{n f} c_{i} \int_{0}^{1}\left\{\frac{\phi_{i}^{e} \phi_{j}^{e}}{\left(\Delta_{2} \xi_{2}+r_{c}\right)}+\frac{\mu}{\Delta_{2}}\left(\phi_{i}^{e^{\prime}} \phi_{j}^{e}+\phi_{i}^{e} \phi_{j}^{e^{\prime}}\right)+\frac{\left(\Delta_{2} \xi_{2}+r_{c}\right)}{\left(\Delta_{2}\right)^{2}} \phi_{i}^{e^{\prime}} \phi_{j}^{e^{\prime}}\right\} h \Delta_{2} d \xi_{2} \\
& +\frac{E_{1}}{\left(1-\mu^{2}\right)} \sum_{j=1}^{n f} \sum_{i=1}^{n f} c_{i} \int_{0}^{1}\left[\left\{\frac{\left(\Delta_{1} \xi_{1}+a\right)}{\left(\Delta_{1}\right)^{2}} \phi_{i}^{p^{\prime}} \phi_{j}^{p^{\prime}}\right\}+\left\{\frac{\phi_{i}^{p} \phi_{j}^{p}}{\left(\Delta_{1} \xi_{1}\right)}\right\}\right. \\
& \left.+\frac{\mu}{\Delta_{1}}\left\{\phi_{i}^{p^{\prime}} \phi_{j}^{p}+\phi_{i}^{p} \phi_{j}^{p^{\prime}}\right\}\right] h \Delta_{1} d \xi_{1}
\end{aligned}
$$




$$
\begin{aligned}
= & \rho \omega^{2} \sum_{j=1}^{n f} \int_{0}^{1}\left\{(\Delta \xi)^{2} \phi_{j}\right\} h \Delta d \xi \\
& -\frac{E-E_{1}}{\left(1-\mu^{2}\right)} \sum_{i=1}^{n f} \int_{0}^{1}\left\{\varepsilon_{r}^{0}\left(\mu \phi_{j}^{p}+\frac{\left(\Delta_{1} \xi_{1}\right)}{\Delta_{1}} \phi_{j}^{p^{\prime}}\right)+\varepsilon_{t}^{0}\left(\phi_{j}^{p}+\mu \frac{\left(\Delta_{1} \xi_{1}\right)}{\Delta_{1}} \phi_{j}^{p^{\prime}}\right)\right\} h \Delta_{1} d \xi_{1} .
\end{aligned}
$$

In the above equation, ()$^{\prime}$ indicates differentiation with respect to normalized coordinates. In equation (28), $\xi$ is normalized global coordinate, $\xi_{1}$ and $\xi_{2}$ are normalized local coordinates in post-elastic and elastic region, $\Delta_{1}=r_{c}$ and $\Delta_{2}=b-r_{c}$. The set of equations given by equation (28) is solved by an iterative scheme as the location of plastic front is not known a priori. Only a brief description of the solution algorithm is presented to keep the volume of this paper within reasonable limit. Due to the same reason, only a validation of plastic limit angular speed obtained by the present method is mentioned in the following paragraph.

\subsection{Solution algorithm}

The required solution of unknown coefficients $\{c\}$ is obtained numerically by using an iterative scheme. To obtain the values of $\varepsilon_{r}^{0}$ and $\varepsilon_{t}^{0}$, to generate the right hand side of equation (28) for a particular load step above elastic limit angular speed, the ratio of $\sigma_{t}$ and $\sigma_{r}$ in each radial coordinate of the complete field is assumed to be same to that of previous load step. As the plastic front originates at the centre of the disk (for solid disk of uniform thickness) at the elastic limit angular speed, with each subsequent increase in the speed above the elastic limit angular speed, plastic front proceeds towards the outer radius. For each load step, the location of plastic front is given a small increment starting from its exact location solved for the previous load step and attainment of von Mises stress at the plastic front location equal to the value of unidirectional yield stress gives the required solution for that load step.

\subsection{Validation}

The non-dimensional angular speed $\Omega$ is defined as $\Omega=\omega b \sqrt{\rho / \sigma_{y}}$. Non-dimensional plastic limit angular speed $\Omega_{2}$ obtained by the present method using $\rho=7850 \mathrm{Kg} / \mathrm{m}^{3}, b=1.0 \mathrm{~m}$ and $\mu=0.3$ is given by 2.0698 , whereas, it's value obtained in Eraslan (2002) is $2 \cdot 1175$. This comparison shows good agreement, thus establishing the validity of the present method. Other material parameter values used for this analysis are same as taken for bar problem.

\section{Conclusion}

In this paper, an approximate elasto-plastic analysis of a solid circular slender bar of various profiles under different loading has been carried out by a numerical method and the formulation is based on the variational principle with assumed displacement field. Linear elastic linear strain hardening type material behaviour has been assumed in the present paper, but the formulation can take up general nonlinear material as well. Normalized load vs displacement plot and normalized load vs plastic front location plots have been presented for various geometric parameters. The location of initiation of elasto-plastic front and its growth are found to be functions of geometry of the bar and loading conditions. To establish the adequacy of the present method the dynamic problem of a taper bar in elasto-plastic region has been 
solved and the results are validated with a commercial FEM package. The method has been established further by formulating a two-dimensional axisymmetric problem and solving a test problem of bidirectional stress field of a rotating uniform disk in post-elastic regime.

The authors are indebted to the anonymous reviewer, whose comments had been instrumental towards improvement of the quality and content of the paper.

\section{List of symbols}

$b \quad$ Outer radius of disk

$c_{i} \quad$ The vector of unknown coefficients

$d_{0} \quad$ Diameter of the bar at the fixed end

$e, p \quad$ Superscripts, correspond to elastic and plastic state

$d_{i} \quad$ Diameter of the bar at the free end

$E, E_{1} \quad$ Elasticity modulus and tangent modulus of the bar material

$[K] \quad$ Stiffness matrix

$h \quad$ Thickness of disk

$I_{0} \quad$ Area moment of inertia of bar at fixed end

$k, n \quad$ Parameters controlling the cross-sectional diameter variation of bar

$L \quad$ Length of the bar

$L_{e} \quad$ Length of the elastic region

$L_{p} \quad$ Length of the plastic region

$L^{\prime} \quad$ Lagrangian

$[M] \quad$ Mass matrix

$n f \quad$ Number of orthogonal functions to approximate displacement field for $u$

$n f^{\prime} \quad$ Number of orthogonal functions to approximate displacement field for $w$

$P \quad$ Load at any instant

$P_{y} \quad$ Load causing yielding

$P_{c} \quad$ Collapse load

$P^{*} \quad$ Normalized load

$r_{c} \quad$ Plastic front location

$r, t \quad$ Subscripts, correspond to radial and tangential direction

$T \quad$ Total kinetic energy

$u \quad$ Displacement field

$u_{1} \quad$ Displacement at the free end

$u^{*} \quad$ Normalized displacement at the free end

$U \quad$ Strain energy

$V \quad$ Potential energy due to external loading

$w \quad$ Transverse displacement

$x^{*} \quad$ Normalized plastic front location

$x_{p} \quad$ Location of yield point for bar

$y \quad$ Coordinate in transverse direction

$\alpha_{i} \quad$ The set of orthogonal functions used as coordinate functions for $w$

$\gamma_{i} \quad$ Set of temporal functions

$\rho \quad$ Density of the bar/disk material 


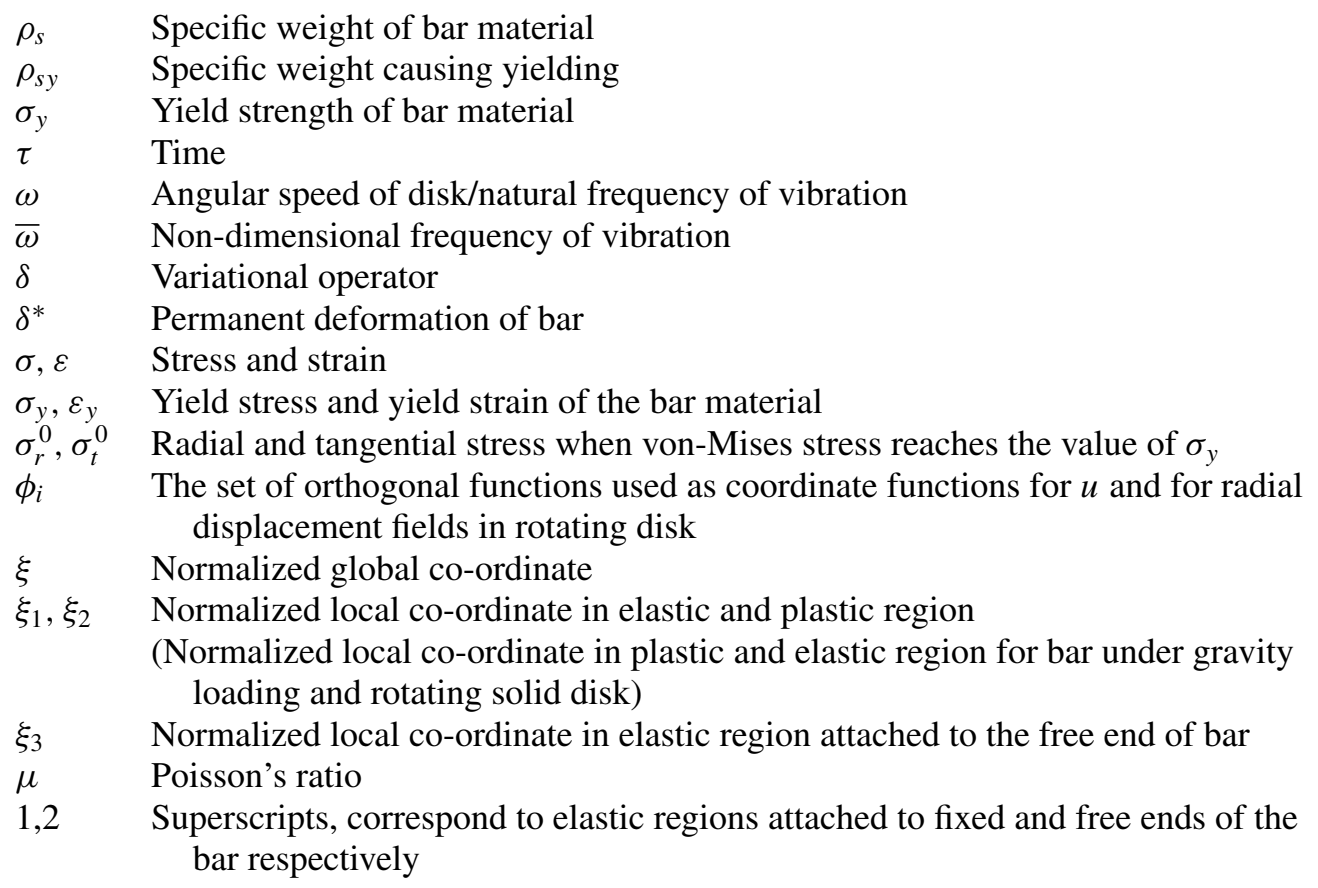

\section{References}

Abdalla H F, Megahed M M, Younan M Y A 2006 A simplified technique for shakedown limit load determination. Nuclear Eng. and Design 237: 1231-1240

Chaudhuri S R 2008 Change in instantaneous eigen properties due to yielding of a structure. J. Sound Vib. 312: 754-768

Eraslan A N 2002 Inelastic deformations of rotating variable thickness solid disks by Tresca and Von Mises criteria. Int. J. Comput. Eng. Sci. 3: 89-101

Gadala M S, Wang J 1998 ALE formulation and its application in solid mechanics. Computer Methods in Appl. Mech. and Eng. 167(1): 33-55

Gang C, Yinghua L, Bingye X 2003 Plastic limit load analysis of defective pipelines. Acta Mechanica Solida Sinica 16(2): 102-109

Gerstmayr J, Irschik H 2003 Vibrations of the elasto-plastic pendulum. Int. J. Non-Linear Mech. 38: $111-122$

Heller B, Kleiner M 2006 Semi-analytical process modelling and simulation of air bending. The Journal of Strain Analysis for Engineering Design 41(1): 57-80

Hill R 1950 The mathematical theory of plasticity (Oxford: Oxford university Press)

Holland D V, Virgin L N, Plaut R H 2008 Large deflections and vibration of a tapered cantilever pulled at its tip by a cable. J. Sound Vib. 310: 433-441

Kim Y J, Oh C K, Myung M S, Park J M 2006 Fully plastic analyses for notched bars and plates using finite element limit analysis. Eng. Fract. Mech. 73: 1849-1864

Mattes H S C, Chimissot F E G 1997 Necking of elasto-plastic rods under tension. Int. J. Non-Linear Mech. 32(6): 1077-1086

Owen D R J, Hinton E 1980 Finite elements in plasticity: Theory and practice (UK: Pineridge Press Limited)

Reddy J N 1984 Energy and variational methods in applied mechanics (USA: John Wiley \& Sons)

Reddy J N 2005 An introduction to non-linear finite element analysis (Oxford: Oxford University Press) 
Saha K N, Misra D, Ghosal S, Pohit G 2005 Nonlinear free vibration analysis of square plates with various boundary conditions. J. Sound Vib. 287: 1031-1044

Shames I H, Dym C L 1995 Energy and finite element methods in structural mechanics (India: New Age International Publishers Limited, First Edition)

Yankelevsky D Z 1999 Elastic-plastic behaviour of a shallow two bar truss. Int. J. Mech. Sci. 41: $663-675$ 